Louisiana State University

LSU Digital Commons

1969

\title{
Application of the Pseudopotential Method to Atomic Scattering.
}

Panayiotis Sophocleous Laghos

Louisiana State University and Agricultural \& Mechanical College

Follow this and additional works at: https://digitalcommons.Isu.edu/gradschool_disstheses

\section{Recommended Citation}

Laghos, Panayiotis Sophocleous, "Application of the Pseudopotential Method to Atomic Scattering." (1969). LSU Historical Dissertations and Theses. 1601.

https://digitalcommons.Isu.edu/gradschool_disstheses/1601

This Dissertation is brought to you for free and open access by the Graduate School at LSU Digital Commons. It has been accepted for inclusion in LSU Historical Dissertations and Theses by an authorized administrator of LSU Digital Commons. For more information, please contact gradetd@lsu.edu. 
This dissertation has been microfilmed exactly as received $70-248$

LAGHOS, Panayiotis Sophocleous, 1940APPLICATION OF THE PSEUDOPOTENTIAL METHOD TO ATOMIC SCATTERING.

The Louisiana State University and Agricultural and Mechanical College, Ph.D., 1969

Physics, atomic

University Microfilms, Inc., Ann Arbor, Michigan 
APPLICATION OF THE PSEUDOPOTENTIAL METHUD TO ATOMIC SCATTERING

\author{
A Dissertation \\ Submitted to the Graduate Faculty of the \\ Lou isiana State University and \\ Agricultural and Mechanical College \\ in partial fulfillment of the \\ requirements for the degree of \\ Doctor of Philosophy \\ in
}

The Department of Physics and Astronomy

by

Panayiot is Sophocleous Laghos

B.Sc. American University of Beirut 1964 May 1969 


\section{ACKNOWLEDGMENT}

The author wishes to express his sincere appreciation to Professor Joseph Callaway for much inspiration and patient guidance throughout this work.

He is indebted to the LSU Computer Research Center for the service which they made available, and to Mrs. Janet Borg for assistance with numerical calculations. He would also like to thank Dr. Neil Kestner of the LSU Chemistry Department for the use of certain molecular integral programs.

Financial assistance received from the "Dr. Charles E. Coates Memorial Fund of the LSU Foundation donated by George H. Coates't for the publication of this work is gratefully acknowledged. 
List of Figures and Figure Captions -...-...- iv

Abstract -

I Introduction -

Il Interatomic Potentials - Brief Literature Review --.-.-- 4

III Elastic Scattering and Inelastic Transitions -..--.-.-.- 10

IV The Pseudopotential for Alkali Atoms -...............- 14

4.a Qualitative Justification - 14

4.b Formulation of the Pseudopotential Method -........- 15

4.c Determination of the Pseudopotential Parameters---- 19

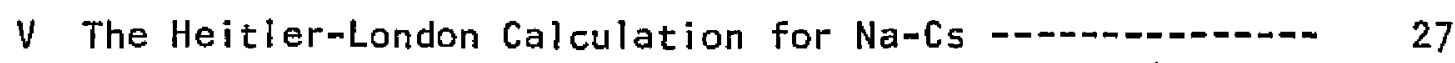

V1 Spin Exchange Cross Sections for $\mathrm{Na}-\mathrm{Cs}$

VII Summary and Conclusion -..........- 50

References -

Vita --10-10 55 


\section{LIST OF FIGURES AND FIGURE CAPTIONS}

F igure

Page

I Examples of purely repulsive potentials.

2 Examples of short range repulsive and long range attractive potentials.

3 Pseudopotential for sodium given by (4.9).

4 Pseudo wave function of a valence electron in the sodium atom obtained-through the use of the pseudopotential. Included for comparison is a reasonable approximation to the true valence electron wave function, ref. 28 .

5 Graphical representation of notation used for the interaction of sodium (A) and cesium (B) atoms when separated by a distance $R$.

6 Coordinate system for two-centre molecular integrals.

$\lambda$ is constant on ellipsoids of revolution, $\mu$ on hyperboioids.

7 Heitler-London singlet and triplet potential energy curves and their difference for Na-Cs. Use of the pseudopotential was made in setting up the interaction Hamiltonian. Included is also the result of the Dalgarno-Rudge formulation (ref. 35) with four term asymptotic coulomb wave functions.

8 Behaviour of the phase $\delta$ and $\sin ^{2} \delta$ as functions of $Z\left(=\alpha_{0} p\right.$, where $p$ is the impact parameter) for a relative velocity of $6.51 \times 10^{4} \mathrm{~cm} / \mathrm{sec},\left(500^{\circ} \mathrm{K}\right)$. 
9 Thermal average of reduced spin exchange cross

sections for $\mathrm{Na}-\mathrm{Cs}$ as a function of system temperature, $\theta$. 49 
ABSTRACT

The empirical pseudopotential method is discussed. Wave functions for the free sodium and cessium atoms which agree well with the exact valence electron functions at large distances are obtained by numerical solution of the wave equation incorporating the pseudopotential. A Heitler-London calculation is made for the lowest singlet and triplet states of the $\mathrm{Na}-\mathrm{Cs}$ system. The difference in energies of these states is used in the calculation of the spin exchange cross section in collisions of sodium and cesium atoms. The scattering phase shifts are calculated in the JWKB approximation, and the cross section computed from them is averaged over a Boltzmann distribution of relative velocities. At a temperature of $500^{\circ} \mathrm{K}$, the averaged reduced spin exchange cross section is $1.48 \times 10^{-14} \mathrm{~cm}^{2}$.

The effect of elastic scattering on the cross section for electronic excitation in the collisions of slow atoms interacting by Van der Waals forces is estimated, and found to be small in usual circumstances. 


\section{INTRODUCTION}

Knowledge of interatomic potentials is of fundamental importance in many problems in physics, astronomy, and chemistry. In the case of atoms with few electrons reasonably accurate Quantum Mechanical calculations from first principles are possible. For heavier atoms, however, great mathematical and computational difficulties arise. In this respect, a class of atoms having only one valence electron and that in a s state, i.e. the alkalies, deserve special consideration. As a first step one can think of the alkalies as one-electron atoms with the single electron moving in a non-coulomb field. The second step is to realize that the effects of electrons in closed shells are not removed entirely (by the first step) because even in low energy problems where only the valence electrons are of real interest, it is still necessary to consider the orghogonality of the valence electron wave function to the wave functions of electrons in closed shells in both of the interacting atoms. Thus it is desirable to remove the core electrons from the problem completely. This is accomplished by the pseudopotential discussed in sec. IV.

When two alkal $\mathbf{i}$ atoms in their ground states approach each other, two spin states are possible, a singlet and a triplet, and each of these has its own potential energy curve. In the present case we are interested in spin exchange scattering which, in the semiclassical approximation, depends on the difference between these two energy curves. 
Moreover, we are interested in atomic interactions at moderately large separations where there is no overlap of the wave functions of core electrons, and we propose to calculate the difference in potentials in the Heitler-London approximation. (See Sec. V).

The Hamiltonian for a system of interacting $\mathrm{Na}$ and $\mathrm{Cs}$ atoms is set up, using the atomic pseudopotentials. The total wave function for the interacting system is approximated by a properly antisymmetrized product of functions of the individual electrons. For these separate functions one uses the wave functions resulting from solving the wave equation for each of the individual free atom (participating in the interaction) making use of the pseudopotential. Undoubtedly this is not exact. For an improved calculation one would have to consider also explicitly the excited states of the atoms. However, it certainly is a reasonable approximation for the lowest states of the quasi-molecule.

The spin exchange cross section, (Sec. VI), for the scattering of alkali metal atoms is determined by the difference in potential energy curves between singlet and triplet states, and since the atomic states involved are s states, this difference will be unaffected by a common Van der Waals potential. It is proper to note at this point that other atoms introduce not only the difficulty of having more electrons but of having them in $p$ or $d$ shells in which case the angular dependent part of the Van der Waals force would have to be taken into consideration.

A thesis on atomic interactions, however, cannot be complete until the effects of elastic scattering (deviations from straight 
line trajectory) are examined. The excitation of an atom in a collision with another atom in a situation in which the dominant interaction is the Van der Waals interaction has been studied. 1,2 It is, therefore, interesting to obtain a quantitative measure of the effect of elastic scattering on the cross section of electronic excitation in the collision of slow atoms interacting by van der Waals forces. ${ }^{3}$ This is done in Sec. 111 . 


\section{INTERATOMIC POTENTIALS - BRIEF LITERATURE REVIEW}

The study of atomic interactions presents us with the difficulties of the many electron problem which we cannot handle exactly but can only treat by approximate methods. In the limit of two atoms approaching each other very slowly, the concept of an interatomic potential energy function, $V(R)$, has become very useful. It has been extensively used in the study of a considerable range of phenomena associated with the equation of state and transport properties of gases and solids. For example, classical statistical mechanics relates $V(R)$ to the temperature-dependent second virial coefficient, $B(T)$, through the equation 4

$$
B(T)=2 \pi N_{0} \int_{0}^{\infty}\left[1-\exp \left(-\frac{V(R)}{K T}\right)\right] R^{2} d R
$$

where $N_{0}$ is Avogardo's number, $R$ is the interatomic separation and $K$ is the Boltzmann constant, with quantum corrections, $\Delta B(T)$ given by

$\Delta B(T)=\frac{h^{2}}{\mu} \frac{N_{o}}{24 \pi K^{3} T^{3}} \int_{0}^{\infty} \exp \left(-\frac{V(R)}{K T}\right)\left(\frac{d V(R)}{d R}\right)^{2} R^{2} d R$

In (2.2) $h$ is Planck's constant, $\mu$ is the reduced mass of the two atoms whose interaction is described by the potential $V(R)$. Also the kinetic theory of gases relates it to the transport properties of gases (coefficients of viscosity $\eta$, diffusion $D$, and heat conductivity $\lambda$ ) through the general integral 4,5 


$$
\begin{array}{r}
\Omega^{(\ell, r)=(2 \pi K T / \mu)^{\frac{1}{2}} \int_{0}^{\infty} \int_{0}^{\pi} \exp \left(-\gamma^{2}\right) \gamma^{2 r+3}\left(1-\cos ^{\ell} \theta\right) \times} \\
\quad l(\gamma, \theta) \sin \theta d \theta d \gamma
\end{array}
$$

with

$$
\gamma^{2}=\frac{1}{2} \frac{\mu v^{2}}{K T}=\frac{E}{K T}
$$

and $\ell=r=T$ for $D$, and $\ell=r=2$ for $\eta$ and $\lambda$.

In $(2.3) V(R)$ enters the equation through the differential crosssection $I(\gamma, \theta)$.

Despite the usefulness of the interatomic potential we have not been able to develop a method simple enough for calculating it, especially at small interatomic separations where the forces are repulsive. For large separations, London ${ }^{6}$ has proved by quantum theory that the interaction potential should be proportional to $-R^{-6}$. The trend has therefore been to invent mathematical models which would be (i) adequate in the region of repulsive as well as attractive forces, ( $i i)$ applicable to a large number of systems and, ( $i$ i)simple enough to be used in calculations.

The various analytic expressions being used contain one, two or more arbitrary parameters which are determined by using these potentials to fit experimental data for gases (e.g.viscosities, virial coefficients), the crystalline state (e.g. equilibrium properties of the crystal like the entropy, energy, and density), etc. This procedure had great successes with systems that are chemically non-reacting (e.g. the inert gases) or having repulsive electronic states (e.g. the ${ }^{3} \Sigma$ state of $\mathrm{Na}_{2}$ ). It has not, however, 
been uniformly successful. For example it is possible to obtain a set of parameters for some form of the potential which would fit experimental data for, say, the second virial coefficient for Argon in its gaseous state but the same set of parameters would give a poor fit to the experimental data for its viscosity. ${ }^{7}$ Furthermore, with chemical interactions a too simple form for the potential is no longer satisfactory because it cannot adequately describe the exchange attractions which depend strongly on the nature of the atoms involved.

In figures 1 and 2 we present some of the types of interatomic potentials used for systems having spherical symmetry. Applications, merits, and regions of applicability of each of these as well as many others have been presented by several authors $4,7-12$ and we will not dwell on them here any further.

At this point it is appropriate to give a word of caution. The whole concept of interatomic potential breaks down if the change in the energy of interaction within a distance of one De Broglie wavelength $(\lambda=h / \mu v)$ is comparable to the separation between this and another potential energy curve ${ }^{13}$. Furthermore, in cases where two potential energy curves approach each other at some interatomic separation that is not too large, so that the interaction is still strong enough to cause transitions, then even slow collisions do not follow a single potential energy curve. 

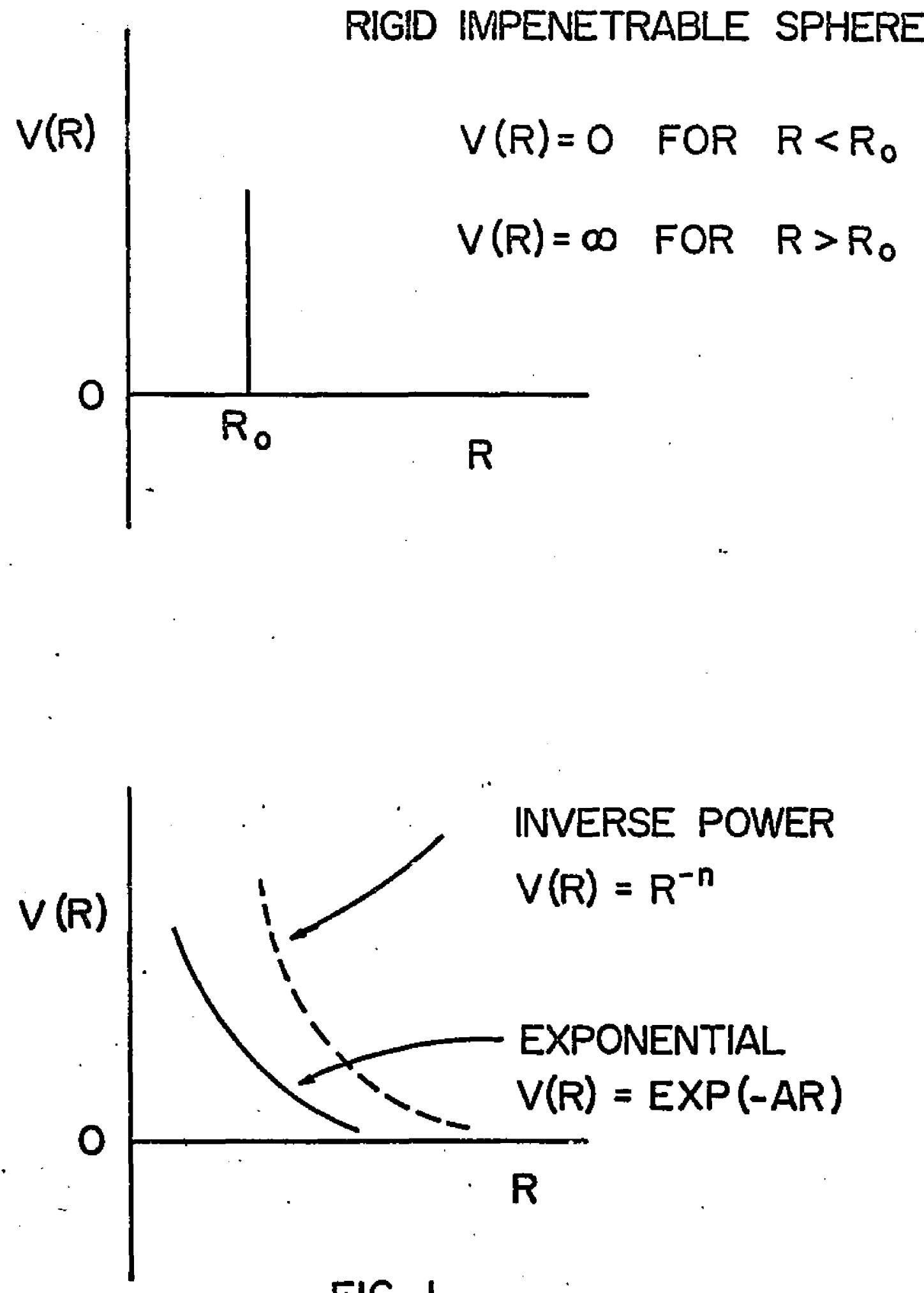

FIG. I . 

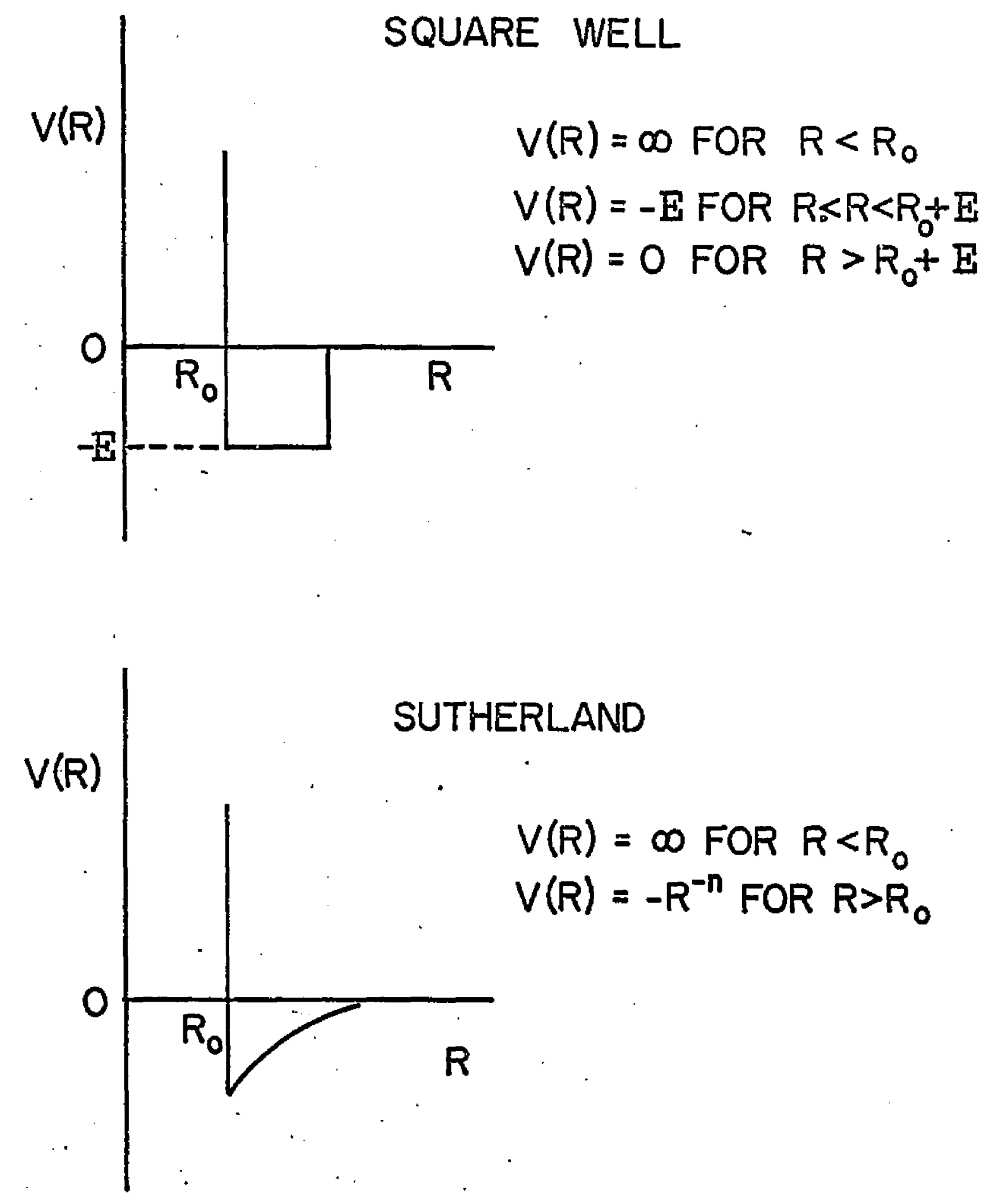

FIG. $2 a$ 

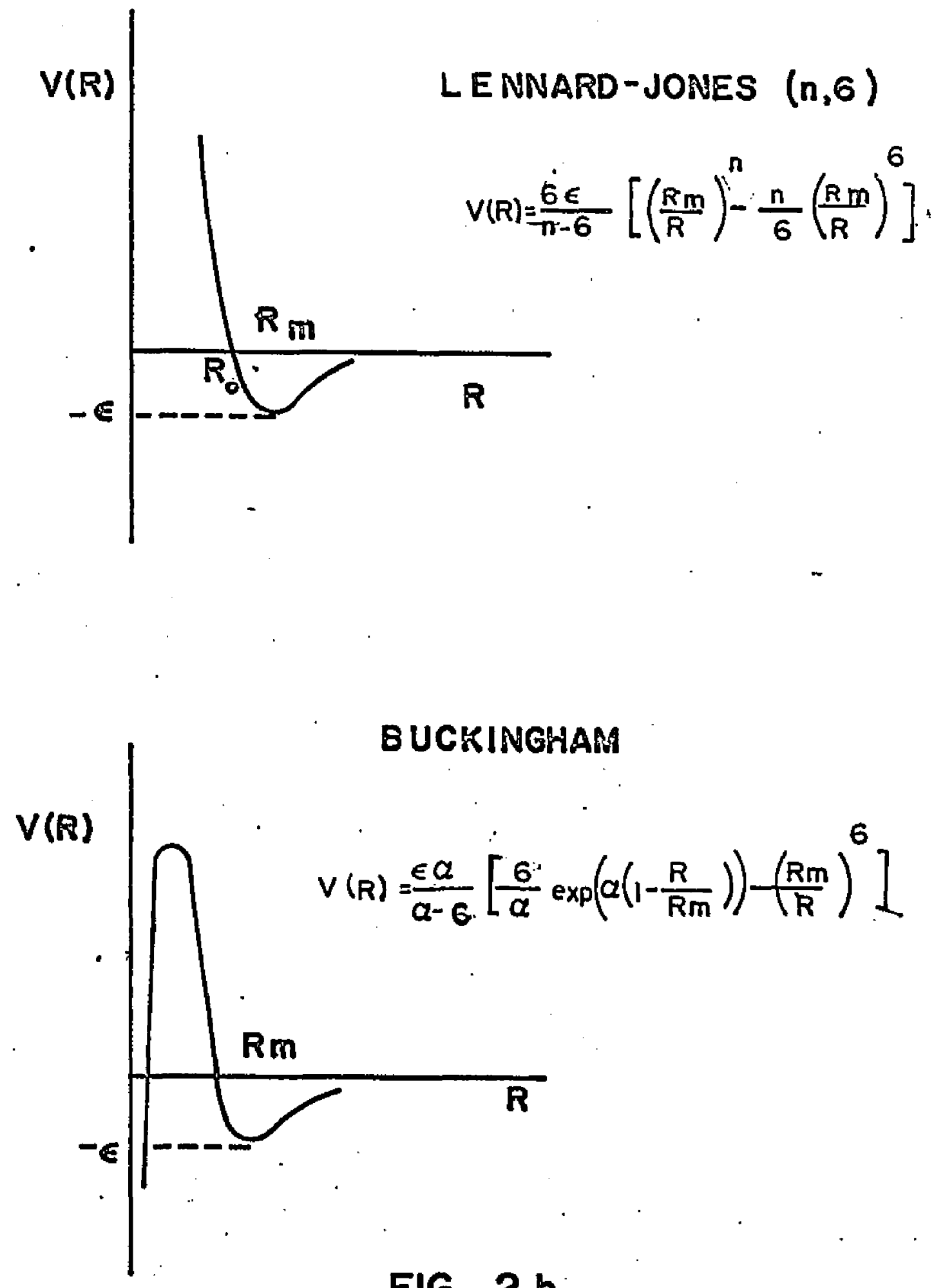

FIG. 2 b 


\section{II ELASTIC SCATTERING AND INELASTIC TRANSITIONS}

The studies considered by Call laway et.al. 1,2 have employed the impact parameter method: the motion of the heavy particle was treated classically, and both the change in speed due to loss or gain of energy during the excitation, and the change in direction of motion due to elastic scattering were ignored.

Although cross sections computed using these methods are in reasonable agreement with experiment, we believe it is important to consider possible corrections to these approximations. ${ }^{3}$ Consider, for example, the reaction

$$
\mathrm{Na}\left({ }^{2} \mathrm{P}_{3 / 2}\right)+\mathrm{Ar} \rightleftarrows \mathrm{Na}\left({ }^{2} \mathrm{P}_{\mathrm{I} / 2}\right)+\mathrm{Ar}
$$

The energy difference between the states is $.002 \mathrm{eV}$. This process has a cross section $\sigma \approx 0.6 \times 10^{-14} \mathrm{~cm}^{2}$ at energies of about $400^{\circ} \mathrm{K}$. If we consider a characteristic length for this reaction $\ell_{0}=(\sigma / \pi)^{\frac{1}{2}}$, the strength of the Van der Wals potential at distance $L_{0}$ is close to the initial kinetic energy. Therefore it is desirable to investigate elastic scattering by the Van der Waals potential.

The theory of the elastic scattering of atoms has been extensively studied by Ford and Wheeler ${ }^{14}$. We will confine our attention here to grazing collisions in which the short range repulsive forces between the atoms are unimportant.

Our approach is based on ref. 1, to which the reader is referred for the notation and for a more complete discussion of the methods 
employed. The transition amplitude is found from an approximate solution of the time dependent Schrodinger equation. The interaction matrix elements are functions of $R$, the distance between the colliding atoms (and of angles also, which we neglect). We consider a scattering potential $V_{s}=-c / R^{6}$. We write $R(t)=\left(p^{2}+v^{2} t^{2}\right)^{\frac{1}{2}}(1+f(t))$. A differential equation for $f$ is readily obtained and solved under the restriction that only terms linear in $c$ are included. The result is:

$f(t)=-c \frac{1+\frac{v^{2} t^{2}}{p^{2}+v^{2} t^{2}}+\frac{3 v t}{2 p \arctan v t / p}}{\mu v^{2} p^{6}\left(1+v^{2} t^{2} / p^{2}\right)}$

Here $\mu$ is the reduced mass of the colliding atoms, $v$ the relative velocity, and $p$ the impact parameter.

The inelastic processes will be described in a two level model. It is assumed that the transition is produced by Van der Waals interactions. We put $\left\langle 1\left|v_{s}\right| 2\right\rangle=q / R^{6},\left\langle 1\left|v_{s}\right| 1\right\rangle=-\left\langle 2\left|v_{s}\right| 2\right\rangle=r q / R^{6}$. Thus $r$, here, measures the ratio of diagonal to off diagonal elements. Assume that the system was originally in state 1. The amplitude for it to be in state 2 at $t=\infty$ is found to be

$$
\left|\alpha_{2}(p)\right|^{2}=\eta^{-2}\left(1-\frac{x^{2} n^{2}}{3 \eta^{2}} \sin ^{2}\left[\left(\frac{b}{p^{5}}+\frac{d}{p^{11}}\right)\left(1-\frac{x^{2}}{6 \eta^{2}}\right]\right.\right.
$$

where $\eta^{2}=1+r^{2}, x=\omega p / v, b=3 \pi q \eta / 8 h v, d=315 \pi q c \eta / 128 \pi \mu v^{3}$. Eq. (3.2) is exact for the model if $\omega=0$. 
The cross section is determined by

$$
\sigma=2 \pi \int_{0}^{\infty}\left|\alpha_{2}(p)\right|^{2} p d p
$$

This integral cannot be done exactly even if $\omega=0$. However, we are interested in terms 1 inear in c. To estimate these, we approximate $\sin ^{2} \xi$ by $1 / 2$ when the argument is larger than a number $k$ of order unity, and by $1 / \xi^{2}$ when $i t$ is smaller than $k$. We choose for $k$ the value 1.18 which yields agreement with the known exact value of $\sigma$, $\sigma(0)$ for $d=0$ and $\omega=0$. In the final expression for $\sigma$ we retain the terms $l$ inear in $c$ and $\omega^{2}$, plus the cross term between them. For simplicity we also set $r=1$. The result is

$$
\begin{gathered}
\sigma=\sigma(0)\left[1+0.142 \frac{\mathrm{c}}{\text { |व } \frac{h}{\mu v \ell_{0}}-0.463\left(\frac{\omega \ell_{0}}{\mathrm{v}}\right)^{2}+}\right. \\
0.588\left(\frac{\omega \ell_{\mathrm{o}}}{\mathrm{v}}\right)^{2} \frac{\mathrm{c}}{\left.\mathrm{T} \mid \frac{\mathrm{h}}{\mu \vee \ell_{\mathrm{o}}}\right]}
\end{gathered}
$$

The significance of this expression is not the specific numbers but the general behaviour of the function. Elastic scattering increases the cross section. This is expected, since the attractive force pulls the colliding particles eloser together and thus increases their interaction. Moreover, we find that the effect depends on the ratio of the wave length for a particle of mass equal to the reduced mass of the pair to the length $\ell_{0}$ characterizing the cross section. This ratio is small for heavy particles except at very low speeds. For $\mathrm{Na}$ - Ar collisions, $\mathrm{h} / \mu \mathrm{v} \ell_{\mathrm{o}}=0.013$ at a speed corresponding 
to $400^{\circ} \mathrm{K}$ (using $\sigma(0)=.66 \times 10^{-14} \mathrm{~cm}^{2}$ ) [1], and even for the relatively unfavourable case of oxygen-hydrogen collisions studied in ref. 2, we have for $\sigma(0)=4.2 \times 10^{-16} \mathrm{~cm}^{2}$ a speed corresponding to $400^{\circ} \mathrm{K}, \mathrm{h} / \mu \mathrm{v} \iota_{\mathrm{o}}=0.22$. The term in eqn. (3.3) involving $\left(\omega \mathrm{o}_{\mathrm{o}} / \mathrm{v}\right)^{2}$ alone is usually of greater importance.

These considerations lead to our principal conclusion that inclusion of elastic scattering will not significantly alter the calculated cross sections for excitation under normal circumstances. 


\section{THE PSEUDOPOTENTIAL FOR ALKALi ATOMS}

\section{a. Qualitative Justification.}

In the alkali atoms one valence electron moves mostly outside a compact core of electrons (in closed shells) which are fairly inactive and this enables us to look at the atoms as one-electron atoms, i.e. we assume that in atomic interactions it is only the valence electron that contributes to the interaction. Nevertheless, the problem is still quite complicated because the presence of these inactive electrons requires that the wave function of the valence electron be orthogonal to the wave functions of electrons in closed shells. The result is that the valence electron wave function must vary rapidly near the nucleus. We are thus faced with an unpleasant situation. We have ignored the presence of the electrons in closed shells and yet (in interactions in which only the valence electron is of interest) we still have to include the effects of the core-electrons wave functions. Is there a way out of this dilemna? Is there a way to remove the effect of the core-electrons wave functions from the problem? This is accomplished by the pseudopotential.

Let us make the following observation. The energy of the lowest state of an electron in a $-e^{2} / r$ potential, that is, the Hydrogen atom, is $-13.6 \mathrm{eV}$. Compare this with the energy of the lowest state of the valence electron of any of the alkal $i$ atoms. In the case of sodium, for example, it is $-5.12 \mathrm{eV}$. This tells us 
right away that the effect of the compact ionic core is a repulsion on the valence electron. This repulsive effect may be attributed to the requirement that the valence electron wave function be orthogonal to the wave functions of the core electrons which, requirement, gives $r i s e$ to additional kinetic energy of the valence electron when it is inside the core.

We are thus led to represent the potential energy, $V(r)$, of the valence electron by an expression of the form

$$
V(r)=-\frac{e^{2}}{r}+F(r)
$$

where $F(r)$ is a repulsive potential. The requirements to be satisfied by $V(r)$ are that it reproduces the known energy levels of the free atom which are determined from spectroscopic data and, for large distances from the nucleus, the valence electron is described by a coulomb wave function.

b. Formulation of the Pseudopotential Method

In this section we present briefly a formulation of the Pseudopotential Method as originally developed by Phillips and Kleinman ${ }^{15}$.

We begin by imagining that we know the 'exact' wavefunction for a valence electron in atomic state $n$. Denote it by $\psi_{n}(r)$. Denote the wave functions for core states by $\psi_{c}(r)$. Our aim is to obtain a ipseudo wave function, $\Phi_{n}(r)$, for the valence ielectron that:will be the' 'smooth part (no radial nodes) of $\psi_{n}(r)$. To this end write 


$$
\psi_{n}(r)=\ddot{\Phi}_{n}(r)+\sum_{c} a_{n c} \psi_{c}(r)
$$

The sum extends over all core states. The requirement that $\psi_{n}(r)$ be orthogonal to all core states $\psi_{c}(r)$, i.e.

$$
\left(\psi_{c}, \psi_{n}\right)=0
$$

gives

$$
a_{n c}=-\left(\psi_{c}, \Phi_{n}\right)
$$

so that $(4.1)$ becomes

$$
\psi_{n}(r)=\Phi_{n}(r)-\Sigma_{c}\left(\psi_{c}, \Phi_{n}\right) \psi_{c}(r)
$$

At this point we assume that $\psi_{c}$ and $\psi_{n}$ are eigenfunctions of the same Hamiltonian, $H$, with eigenvalues $E_{c}$ and $E_{n}$, respectively.

$$
H \psi_{\mathrm{c}}=\mathrm{E}_{\mathrm{c}} \psi_{\mathrm{c}}
$$

and,

$$
H \psi_{n}=E_{n} \psi_{n}
$$

To obtain an equation satisfied by $\bar{\Phi}_{n}$, substitute (4.4) into (4.5b) and use (4.5a) to get

$$
H \Phi_{n}-\Sigma_{c}\left(\psi_{c}, \Phi_{n}\right)\left(E_{c}-E_{n}\right) \psi_{c}=E_{n} \Phi_{n}
$$

Write the Hamiltonian as $H=T+V$, where $V$ is the real potential, and use it to reduce the above equation to

$$
\left(T+V_{p}\right) \Phi_{n}(r)=E_{n} \Phi_{n}(r)
$$


where

$$
V_{p} \Phi_{n}(r) \equiv V \Phi_{n}-\sum_{c}\left(E_{c}-E_{n}\right)\left(\psi_{c}, \Phi_{n}\right) \psi_{c}(r)
$$

$v_{p}$ is the desired pseudopotential, and (4.6a) is the desired equation satisfied by the pseudo wave function $\Phi_{n}$. Let us rewrite (4.5b), with $H=T+V$, to get

$$
(T+V) \mho_{n}(r)=E_{n} \psi_{n}(r)
$$

Notice the very important comparison between (4.7) and (4.6a). In order to go from the 'exact' wave equation to $(4.6 a)$ we replace the real potential $V$ by the pseudopotential $V_{p}$ and the 'exact' wave function $\psi_{n}$ by the pseudowave function $\Phi_{n}$, but retain the same eigenvalue.

Let us look at (4.6a) in a little detail. It is evident that $v_{p}$ is an energy-dependent non-local operator and so $(4.6 a)$ becomes an integro-differential equation whose solution must be obtained self consistently. We want to try to avoid this laborious task. Divide both sides of (4.6b) by $\Phi_{n}$." The resulting term $\psi_{c}{ }^{\prime} \Phi_{n}$ we approximate by the simple function $e^{-\beta r} / r$, a form easily suggested if one approximates $\psi_{c}$ and $\Phi_{n}$ by Slater orbitals. As for the energy difference $\left(E_{c}-E_{i}\right)$ one could probably replace it by some average wich would not be a poor approximation if variations of $\left(E_{c}-E_{n}\right)$ were small enough to be neglected.

To avoid all the above we will adopt the Hellmann type pseudopotential ${ }^{16}$.

$$
V_{p}(r)=-\frac{e^{2}}{r}+Q e^{-\beta r} / r
$$


The pseudopotential parameters $Q$ and $\beta$ introduced above are, in effect, a measure of the strength and range of the repulsive part of the potential, respectively. They pertain to the specific free atom one is interested in and will have to be determined from spectroscopic data. (From here on we drop the subscript $p$ from $v_{p}$ ).

This same form of the pseudopotential has been applied to other. molecular problems by Preuss 17 , to the calculation of ground state energies of complex atoms 18,19 , and to problems in solid state physics $^{20-23}$. Other forms of the pseudopotential also exist. Asheroft ${ }^{24}$ has used the form

$$
\begin{array}{rlrl}
V(r) & =-\frac{z e^{2}}{r} & & r>R_{\text {core }} \\
& \sim 0 & r<R_{\text {core }}
\end{array}
$$

Where $R_{\text {core }}$ is some effective core radius, essentially the ionic radius, and has used it for calculations of resistivities, phonon spectra and phase shifts in alkali metals. Zapol et.al. ${ }^{25}$ have used a similar form which is continuous within certain ranges of $r$,

$$
V(r)=-\frac{A_{i}}{r} \quad R_{i} \leq r \leq r_{i+1}(i=0,1, \ldots, k)
$$

to calculate energy spectra and oscillator strengths for monovalent atoms. The arbitrary parameters in the potential were determined by requiring that some of the eigenvalues coincided with the known experimental ones. A different type of pseudopotential, based on the Thomas-Fermi model, proposed by Gombas ${ }^{26}$ in his recent discussion of the subject of pseudopotentials in atomic systems has been 
applied by Bayliss ${ }^{27}$ for calculating approximate interatomic. potentials for ground and low-lying excited states of diatomic systems of alkali-metal noble gas atoms. In closing this section let us point out that in using our freedom for the choice of the repulsive part of the pseudopotential our expression has the advantage of simplicity (with respect to Gombas), continuity (at:(with respect to Zapol et al.) and is simply related to the basici: derivation. It does have the disadvantage of being strong and rapidly varying in the core region, which means that care must be taken in numerical work, as we shall see.

c. Determination of the Pseudopotential Parameters

The units employed are: $e^{2}=2, m=\frac{1}{2}, h=1$ (energy unit $=$ ( Ryd.)

The expression for the pseudopotential, (4.8), is

$$
V(r)=-\frac{2}{r}+Q \exp (-\beta r) / r
$$

and the parameters to be calculated are $Q$ and $\beta$. This is to be done for sodium and cesium. We will treat the sodium case in detail and give only the results for cesium. This will make the procedure used more specific and understandable.

The sodium atom in its ground state has the $1 s, 2 s$, and $2 p$ shells filled. Its valence electron is in the 3 s state, Let us see how the variational method can be used. Denote the (properly normalized) radial parts of the valence electron wave functions for the $3 s$ and $3 p$ states, respectiveiy, by 


$$
\begin{aligned}
& R_{3 s}(r)=\left(\epsilon_{1}^{3} / \pi\right)^{\frac{1}{2}} \exp \left(-\epsilon_{1} r\right) \\
& R_{3 p}(r)=\left(\epsilon_{2}^{5} / 3 \pi\right)^{\frac{1}{2}} r \exp \left(-\epsilon_{2} r\right)
\end{aligned}
$$

with (known) eigenvalues $E_{3 s}$ and $E_{3 p}$. Write the Schrodinger equation for these two states using (4.9) and calculate $E_{35}$ and $E_{3 p}$ in terms of $\epsilon_{1}$ and $\epsilon_{2}$ (and also $Q$ and $\beta$ ). The condition that the energy be a minimum requires $\partial \mathrm{E}_{3 \mathrm{~s}} /{ }_{\epsilon_{1}}=0$ and $\partial \mathrm{E}_{3 \mathrm{p}} / \mathrm{\partial}_{\epsilon_{2}}=0$. This gives enough equations to solve for $Q$ and $\beta$.

The unquestioned merit of this procedure is that it is straightforward and the algebra involved is very easy to handle specially when the trial wave functions, $R(r)$, are simple. Because of this many authors have been using this procedure. Callaway ${ }^{22}$ points out, however, that pcor approximations to the true wave functions for such a type of pseudopotential do not allow the electron to avoid the strongly repulsive core (as is found to occur when the Schrodinger equation is solved) and the result is a too soft repulsive part of the pseudopotential.

The method we used in the present work goes as follows ${ }^{23}$. The radial part of the wave equation is

$$
\frac{d^{2}}{d r^{2}} \Phi_{n \ell}+\left[E_{n \ell}-v-\frac{\ell(\ell+1)}{r^{2}}\right] \Phi_{n \ell}=0
$$

where $E_{n \ell}$ is the known experimental value for the free atom nl state; and $V$ is the pseudopotential with trial values for $Q$ and $\beta$. Far out in the Coulomb region the repulsive part of the pseudopotential 
is negligible, that is, $V=-\frac{2}{r}$ and, in this region, the radial wave equation becomes

$$
\frac{d^{2} P_{n \ell}}{d r^{2}}+\left[E_{n \ell}+\frac{2}{r}-\frac{\ell(\ell+1)}{r^{2}}\right] P_{n \ell}=0
$$

The solution to (4.11) we know. It is the expansion

$$
P_{n \ell}=r^{s} e^{-r / s} \sum_{m} \alpha_{\ell, m} r^{-m}
$$

where $s=\left(-E_{n \ell}\right)^{-\frac{1}{2}}$ and the coefficients $\alpha_{\ell, m}$ satisfy the equation

$$
\alpha_{\ell, m}=[\ell(\ell+1)-(s+1-m)(s-m)] \alpha_{\ell, m-1} /(2 m / s)
$$

Now, for a prescribed state, say the 3s-state of sodium (i.e. $\left.E_{n \ell}=E_{3 s}, \ell=0\right)$, we integrate $(4.10)$ outwards into the Coulomb region (where the wave function is the known $P_{35}$ ) and then vary $Q$ and $\beta$ until we can reasonably match the logarithmic derivatives of $\Phi_{3 s}$ and $\beta_{3 s^{\circ}}$ In practice one holds $Q$ fixed and varies $\beta$. However, this set of values $(Q, \beta)$ is not unique, and one obtains a curve $Q_{1}(\beta)$, say, in the $Q-\beta$ plane, for which solutions are obtained. Let us pause for a moment and ask the question: How can we make the best use of the freedom given to us as to the choice of the

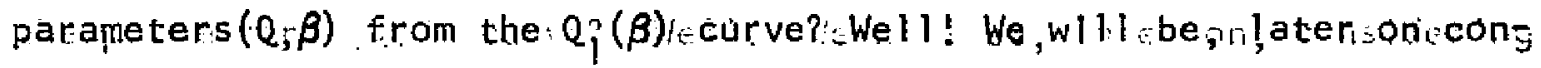
sidering interactions tbetween two (alkalị) atoms Even though we assume that the electrons in closed ghells remaln inert it is not true that 
the valence electron will remain in the s state. That is, the oneelectron atom will be polarized to some degree and to an extent we can take account of this polarization right now, by allowing the pseudopotential to describe the interaction of the valence e with its parent ion when in its first excited state, the p-state. This we accomplish by repeating the above process (in the case of sodium) for the $3 p$ state resulting in a new, $Q_{2}(\beta)$, curve. The intersection of the curves identifies a point at which both states are fit by the same potential, and thus specifies the parameters.

For sodium the $3 \mathrm{~s}$ and the $3 \mathrm{p}$ states were used and an intersection of the two $Q(\beta)$ curves was obtained without difficulty. For cesium the $6 \mathrm{~s}$ and the $6 \mathrm{p}$ states were used. No intersection of the $Q(B)$ curves was found in this case and a set of parameters from the $Q(\beta)$ curve of the $6 s$ state was chosen arbitrarily. The error produced in the $6 p$ state energy was .016 Ryd or $8.8 \%$. The pseudopotential parameters adopted for the work to follow are 1 isted in table $I$. In the case of sodium the results are in good agreement with those of reference 23 .

Table 1. Pseudopotential parameters for Sodium and Cesium The calculated and actual energies for the $3 \mathrm{~s}$ and $3 \mathrm{p}$ states of sodium and the $6 \mathrm{~s}$ and $6 \mathrm{P}$ states of cesium are given

\begin{tabular}{|c|c|c|c|c|c|c|}
\hline Atom & $Q$ & $\beta$ & $\mathrm{E}_{\mathrm{calc}}(\mathrm{s})$ & $E_{\text {exp }}(s)$ & $\mathrm{E}_{\mathrm{calc}}(\mathrm{p})$ & $E_{\text {exp }}(p)$ \\
\hline $\mathrm{Na}$ & 20.43 & 2.0475 & -.3777 & -.377726 & -.2231 & -.223102 \\
\hline Cs & 25.00 & 1.4239 & -.2862 & -.286181 & -.1972 & -.181072 \\
\hline
\end{tabular}


In order to make use of existing molecular integral programs, the calculated s-state pseudo wave functions were fit by a linear combination ( 5 terms) of slater type orbitals, $X_{n}(r, \zeta)$, defined by

$$
x_{n}(r, \zeta) \equiv(2 \zeta)^{n+\frac{1}{2}}((2 n) !)^{-\frac{1}{2}} r^{n-1} \dot{e}^{-\zeta r}
$$

and individually normal ized so that

$$
\int x_{n}^{2}(r, \zeta) r^{2} d r=1
$$

In each case, we write for the normalized total pseudo wave function

$$
\psi=(4 \pi)^{-\frac{1}{2}} \sum_{i=1}^{5} \alpha_{i} x_{n_{i}}\left(r, \zeta_{i}\right)
$$

The expectation value of the free atom Hamiltonian with the analytic pseudo wave function (4.16) along with the slater orbital parameters $n_{i}, \alpha_{i}, \zeta_{i}$ are given in table 2 .

Table 2:. Slater orbital parameters and energies for Sodium and Cesium. See equations $(4.14)$ and $(4.16)$ for definition of the parameters.

\begin{tabular}{ccccccc}
\hline & & \multicolumn{2}{c}{ Sodium } & & \multicolumn{2}{c}{ Cesium } \\
& $n_{i}$ & $\alpha_{i}$ & $\zeta_{i}$ & $n_{i}$ & $\alpha_{i}$ & $\zeta_{i}$ \\
$i$ & 1 & .162795 & .48778 & 1 & .000096 & .12008 \\
2 & 1 & -.942629 & 1.60674 & 1 & -.470277 & .75668 \\
3 & 1 & .801495 & 1.75614 & 1 & .053886 & 3.21463 \\
4 & 2 & .926946 & .65373 & 2 & 1.257517 & .53765 \\
5 & 2 & .003845 & 4.57142 & 2 & .132458 & 2.28540 \\
& Energy & -.377625 & & \multicolumn{2}{c}{-.286169} \\
\hline
\end{tabular}


In figure 3 we plot the pseudopotential for sodium, and in figure 4 we plot the corresponding pseudo wave function. Notice that inside the core the pseudo wave function differs from the true valence electron wave function, in that in the case of the lowest valence state, it is nodeless. For the sake of comparison we show in figure 4 a reasonable qpproximation to the true valence electron wave function for sodium ${ }^{28}$. 


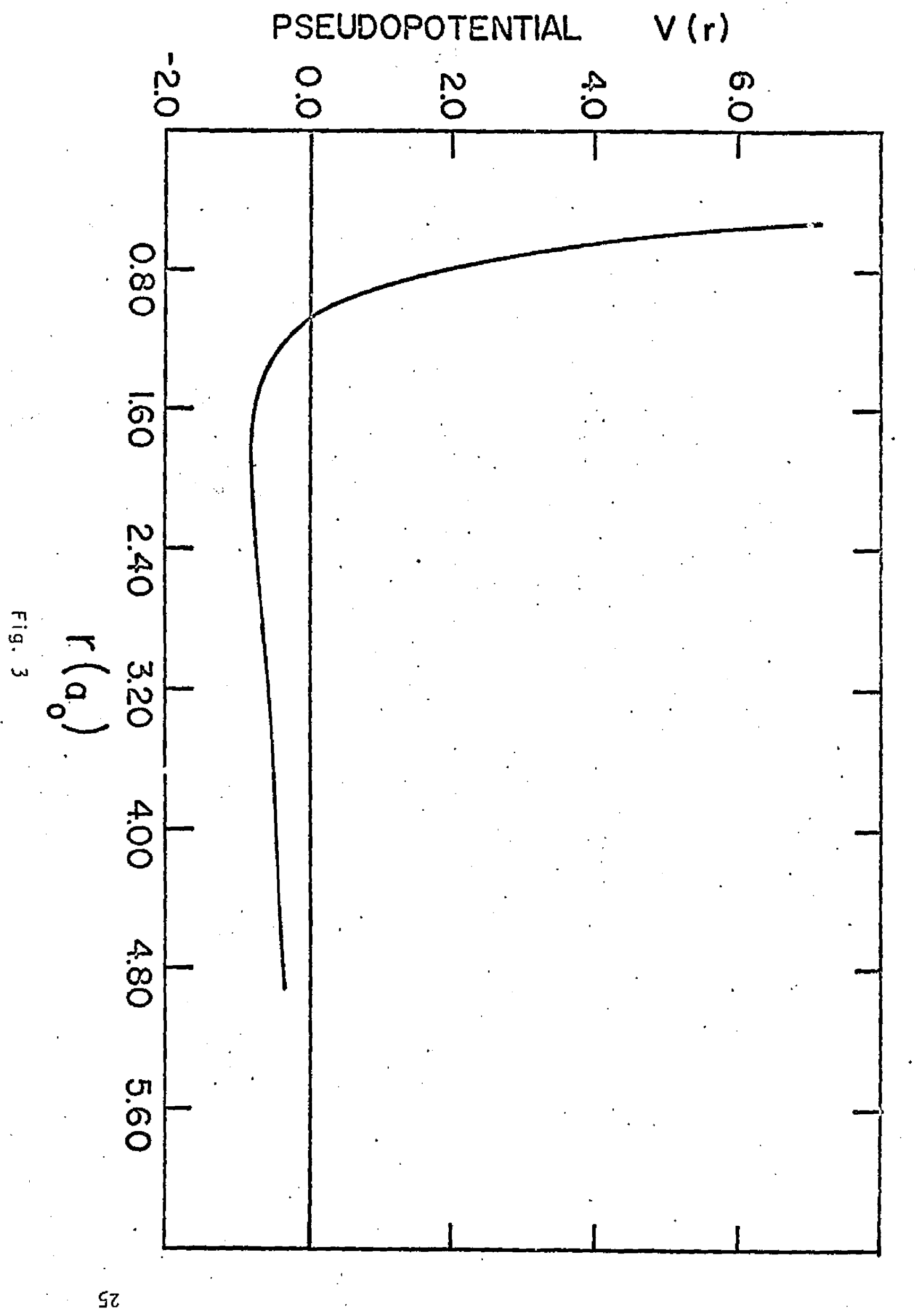




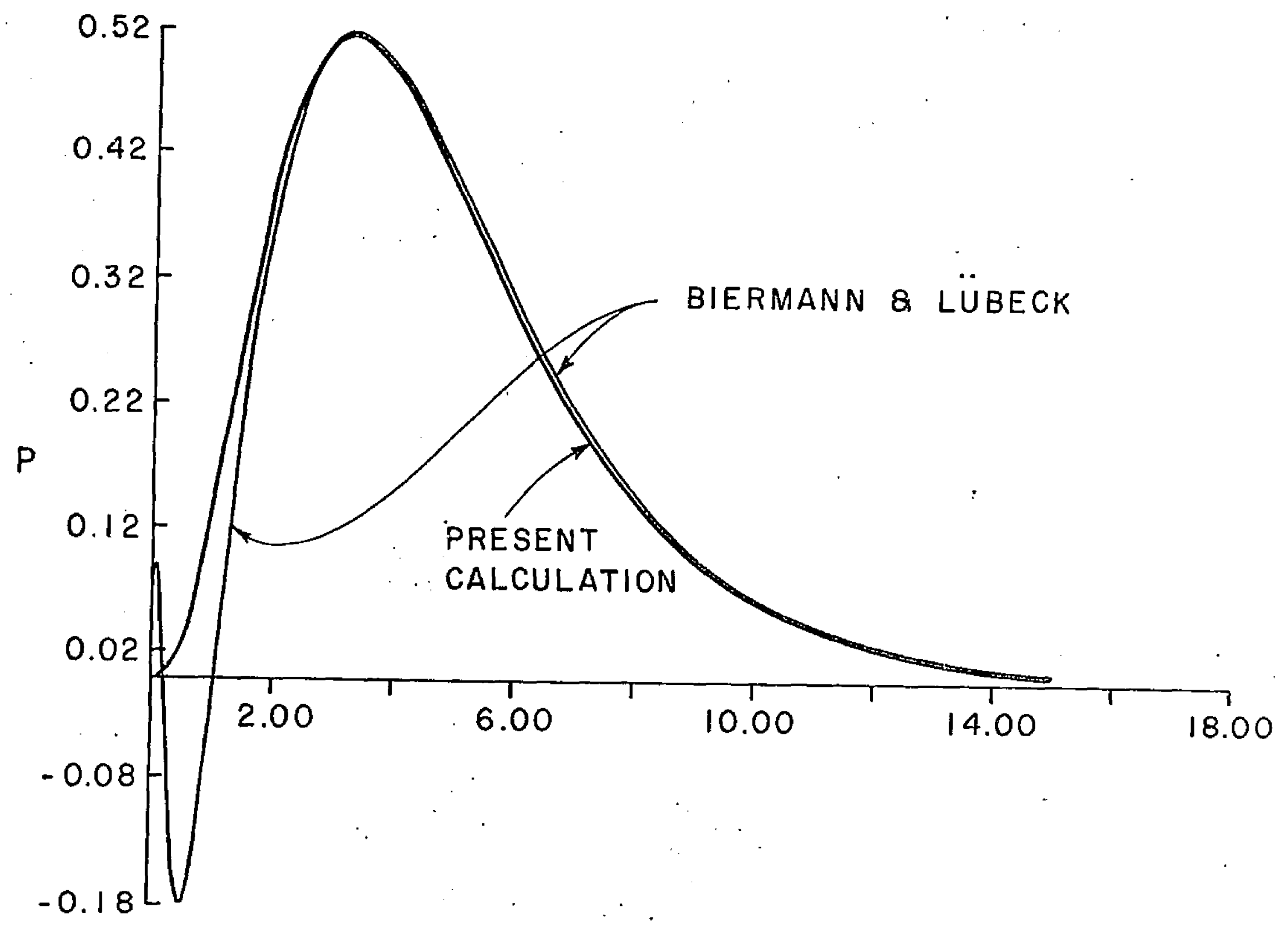


$\checkmark$ THE HEITLER-LONDON CALCULATION FOR Na-Cs.

The Heitler-London method was developed right after the invention of wave mechanics by Schrödinger, in an attempt to get an approximate solution to one of the simplest many electron problems, the $\mathrm{H}_{2}-$ molecule $e^{29}$.

Herring and Flicker have shown that the Heitler-London approximation is incorrect at very large interatomic separations where it predicts, erroneously, that the triplet state should lie below the singlet state in $\mathrm{H}_{2}{ }^{30}$. This crossing of the potential energy curves occurs at an interatomic separation $R_{c}=49.5 a_{0} \cdot$ In their work, Herring and Flicker were able to obtain an exact asymptotic expression for the singlet-triplet energy difference in $H_{2}$. Their result is proportional to $\mathrm{R}^{5 / 2} \mathrm{e}^{-2 \mathrm{R}}$ with leading corrections of order $O\left(R^{2} e^{-2 R}\right)$. In the range of moderately large distances, the leading term in the exact series obtained by Herring and $\mathrm{Fljcker}$ and the results of the standard Heitler-London calculation are in good agreement. It is not possible to ascertain the accuracy of the Heitler-London method in view of the lack of knowledge of higher terms in the exact asymptotic series.

Two reasons make us believe that we are justified in using the Heitler-London method in our present work. (i)- In the Na-Cs system the wave functions die off much slower than in the $\mathrm{H}-\mathrm{H}$ problem, and a crossing of the potential energy curves that one might expect would have to occur at extremely large distances, larger than in the case of hydrogen $\left(R_{c}=49.5 a_{0}\right)$. 
(ii)- The region of interest in the $\mathrm{Na}-\mathrm{Cs}$ system is that of moderately large distances. In view of the preceding observations the HeitlerLondon method should be accurate in this region and, furthermore, because of (i) this region should extend to very large separations beyond which one would get but negligible contributions.

We have made a Heitler-London calculation of the energies of the lowest singlet and triplet states of the Na-Cs system. Only a brief outline of the calculation will be given here since this is now a standard method presented in various text books 3 ! :

Denote the sodium and cesium atoms by $A$ and $B$, respectively, and assign, arbitrarily, electron 1 to atom $A$ and electron 2 to atom $B$. When the atoms are separated by a distance $R$, the Hamiltonian for the interacting system is, in the present approximation,

$$
Z=H_{A}(1)+H_{B}(2)+V_{B}(1)+V_{A}(2)+V(1,2)+V_{A B}
$$

where

$$
\begin{aligned}
H_{A}(1)= & T(1)+V_{A}(1) \text { is the free } N a \text { atom Hamiltonian } \\
H_{B}(2)= & T(2)+V_{B}(2) \text { is the free } C s \text { atom Hamiltonian } \\
V_{x}(i)= & -\frac{2}{r_{i x}}+Q_{x} \text { exp }\left(-\beta_{x} r_{i x}\right) / r_{i x} \text { is the interaction } \\
& \text { between ion } \times(A \text { or } B) \text { and electron } i(1 \text { or } 2) . \text { This } \\
& \text { is, of course, the pseudopotential and the parameters } \\
& Q \text { and } \beta \text { are those given in table } I .
\end{aligned}
$$




$$
\begin{aligned}
& V(1,2)=\frac{2}{r_{12}} \text { is the Coulomb repulsion between electrons } \\
& 1 \text { and } 2 \text {, and }
\end{aligned}
$$

$V_{A B}=\frac{2}{R}$ is the interaction between the two ions. Here we assume the ions to be spherical and not interpenetrating. This will be the case for not too small internuclear distances $R$.

In figure 5 we show graphically the interacting system for some fixed distance $R$. Now, recall the pseudo wave function given by equation $(4.16)$ and write it as

$$
a(1) \equiv(4 \pi)^{-\frac{1}{2}} \sum_{i=1}^{5} \alpha_{i} x_{n_{i}}\left(r_{1 A}, \zeta_{i}\right)
$$

$1 t$ describes the motion of electron $i$ about atom $A$. Without any confusion and similar meaning as above we may write

$$
\begin{aligned}
& \mathrm{b}(2) \equiv(4 \pi)^{-\frac{1}{2}} \sum_{i=1}^{5} \beta_{\mathrm{i}} x_{n_{i}}\left(r_{2 B}, \eta_{\mathrm{i}}\right) \\
& \mathrm{a}(2) \equiv(4 \pi)^{-\frac{1}{2}} \sum_{i=1}^{5} \alpha_{\mathrm{j}} x_{n_{i}}\left(r_{2 A}, \zeta_{i}\right) \\
& \mathrm{b}(1) \equiv(4 \pi)^{-\frac{1}{2}} \sum_{i=1}^{5} \beta_{i} x_{n_{i}}\left(r_{i B}, \eta_{i}\right)
\end{aligned}
$$

All the parameters in (5.2) are defined by equations $(4.14)-(4.16)$ and are found in table 2. (Here we have used different symbols for each atom). The overlap integral, denoted by $S$, is defined as 


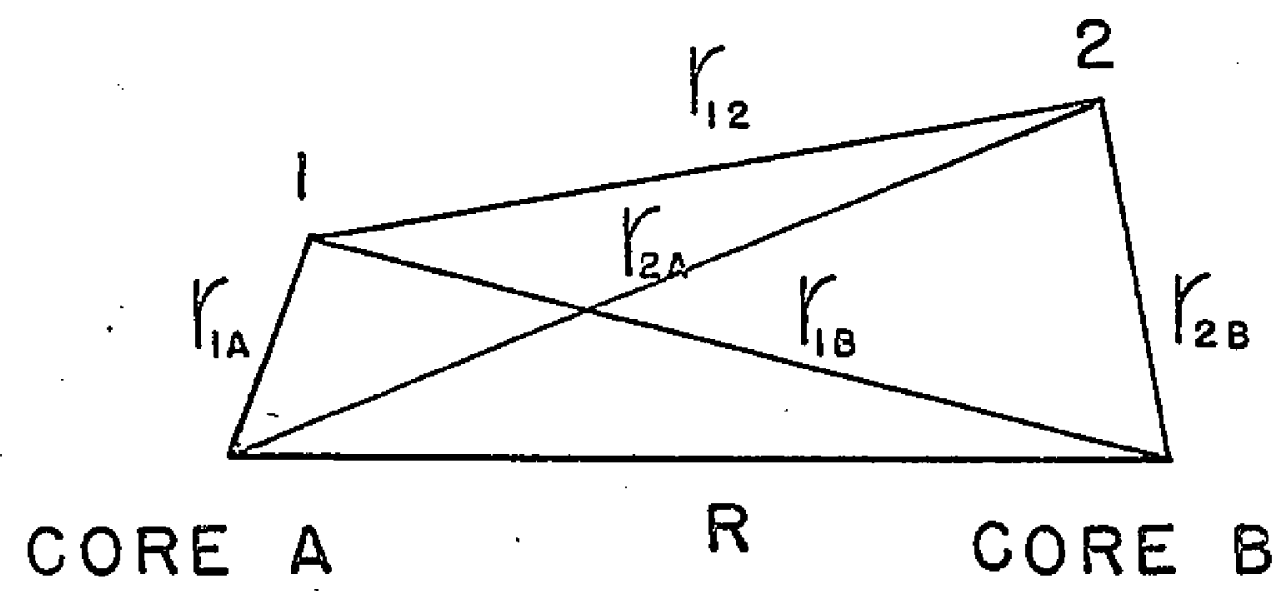

FIG. 5 


$$
s=\langle a(1) \mid b(1)\rangle
$$

The normalized singlet, $s$, and one of the triplet, $t$, states are described by

$$
\underset{t}{\Psi_{s}}=\left[2\left(1 \pm s^{2}\right)\right]^{-\frac{1}{2}}[a(1) b(2) \pm a(2) b(1)]
$$

The Heitler-London energies for the signlet and one of the triplet levels are

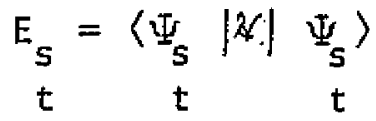

Use of (5.1) and (5.4) in (5.5) yields

$$
E_{s}=\epsilon_{o}(A)+\epsilon_{o}(B)+\frac{V_{c} \pm V_{e x}}{\left(1 \pm s^{2}\right)}
$$

where $\epsilon_{0}(A)$ and $\epsilon_{0}(B)$ are the free sodium and cesium atom eigenvalues, respectively, satisfying

$$
\begin{aligned}
& H_{A}(1) a(1)=\epsilon_{0}(A) a(1) \\
& H_{B}(2) b(2)=\epsilon_{0}(B) b(2)
\end{aligned}
$$

and their values are given in table 2. Here $V_{c}$ and $V_{e x}$, ordinarily referred to as the coulomb or direct and exchange integral, respectively, are 


$$
\begin{aligned}
& v_{c}=\left\langle a(1) b(2)\left|v_{B}(1)+v_{A}(2)+v(1,2)+v_{A B}\right| a(1) b(2)\right\rangle \\
& v_{e x}=\left\langle a(1) b(2)\left|v_{B}(1)+v_{A}(2)+v(1,2)+v_{A B}\right| a(2) b(1)\right\rangle
\end{aligned}
$$

In table 3 we give the various integrals that arise when (5.7) and (5.8) are evaluated. We use, as closely as posible the notation of reference 31 . The evaluation of these integrals is very lengthy, cumbersome, and time consuming, mostly because our wave functions are 1 inear combinations of several terms, (eqn. 5.2). The most difficult are the two centre integrals. One then introduces elliptical coordinates $32,33 \lambda, \mu, \varphi$ as shown in fig. 6 .

Then

$$
\begin{aligned}
& \lambda=\left(r_{A}+r_{B}\right) / R \quad 1 \leq \lambda \leq \infty \\
& \mu=\left(r_{A}-r_{B}\right) / R \quad-1 \leq \mu \leq 1
\end{aligned}
$$

The third coordinate, $\varphi$, is the angle of rotation about the axis $R$ joining the two atoms. The element of volume in terms of these coordinates is

$$
\mathrm{d} \tau=(\mathrm{R} / 2)^{3}\left(\lambda^{2}-\mu^{2}\right) \mathrm{d} \lambda \mathrm{d} \mu \mathrm{d} \varphi
$$

In table 4 we give numerical values of the integrals $S, d_{B}$, $J_{A}, K_{B}, K_{A}, J^{\prime}, K^{\prime}, K_{B}{ }^{\prime}, K_{A}{ }^{\prime}, J_{B}{ }^{\prime}, J_{A}{ }^{\prime}$ defined in table 3 , of $V_{C}$ and $V_{\text {ex }}$ defined by equations (5.7) and (5.8), and of $E_{S}$ and $E_{t}$ 
Table 3. Integrals met in the Heitler-London calculation for Na-Cs.

$$
\langle a(1) \mid b(1)\rangle=s
$$

one-electron two-centre overlap

$\left\langle a(1)\left|-\frac{1}{r_{18}}\right| a(1)\right\rangle=J_{B}$

$\left\langle b(2)\left|-\frac{1}{r_{2 A}}\right| b(2)\right\rangle=J_{A}$

one-electron one-centre coulomb

$\left\langle a(1)\left|-\frac{1}{r_{1 B}}\right| b(1)\right\rangle=K_{B}$

$\left\langle a(2)\left|-\frac{1}{r_{2 A}}\right| b(2)\right\rangle \Rightarrow K_{A}$

one-electron two-centre reasonance

$\left\langle a(1) b(2)\left|\frac{1}{r_{12}}\right| a(1) b(2)\right\rangle=J \cdot \quad$ two-electron two-centre coulomb

$\left\langle a(1) b(2)\left|\frac{1}{r_{12}}\right| a(2) b(1)\right\rangle=K^{\prime} \quad$ two-electron two-centre exchange

$\left\langle a(1)\left|\frac{e^{-\beta_{B} r_{I B}}}{r_{i B}}\right| b(1)\right\rangle=K_{B}^{\prime}$

$\left\langle b(2)\left|\frac{e^{-\beta_{A} r_{2 A}}}{r_{2 A}}\right| a(2)\right\rangle=K_{A}^{\prime}$

$\left\langle a(1)\left|\frac{e^{-\beta_{B} r_{1 B}}}{r_{1 B}}\right| a(1)\right\rangle=J_{B}^{\prime}$

$\left\langle b(2)\left|\frac{e^{-\beta_{A} r_{2 A}}}{r_{2 A}}\right| b(2)\right\rangle \geq J_{A}{ }^{\prime}$

given by equation (5.6), as functions of $R$, as obtained through the use of existing standard molecular integral programs. 


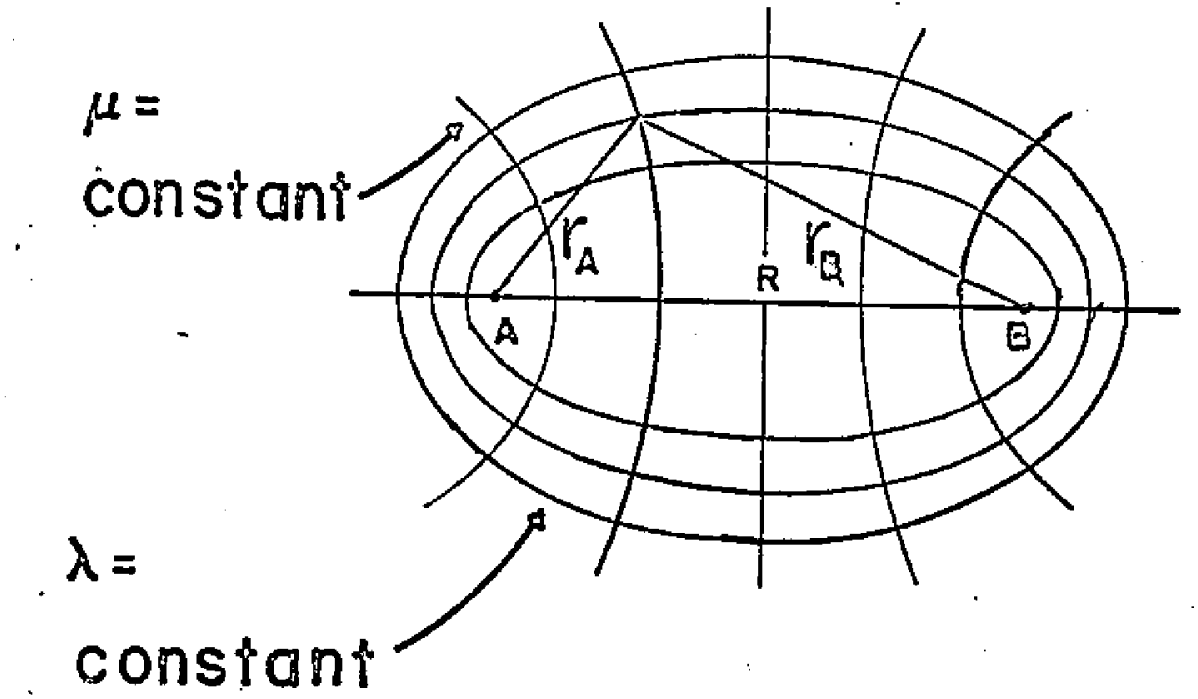

FIG. 6 
Table 4. Numerical values of the integrals met in the Na-Cs Heitler-London calculation.

\begin{tabular}{|c|c|c|c|c|c|c|c|c|}
\hline $\mathrm{R}$ & $\mathrm{S}$ & $J_{B}$ & $J_{B}^{\prime}$ & $J_{A}$ & $J_{A}^{\prime}$ & $\mathrm{J}^{\prime}$ & $K_{B}$ & $K_{B}^{\prime}$ \\
\hline 3.00 & .81780 & -.25900 & .01201 & -.20923 & .00334 & .17594 & -.19515 & .00384 \\
\hline 4.00 & .74244 & -.22379 & .00698 & -.19451 & .00288 & .16595 &.-16950 & .00296 \\
\hline 5.00 & .65562 & -.19089 & .00342 & -.17558 & 10019. & .15448 & -.14167 & .00210 \\
\hline 6.00 & .56201 & -.16355 & .00150 & -.15617 & .00108 & .14227 & -.11407 & .00139 \\
\hline 7.00 & .46755 & -.14181 & .00061 & -.13844 & .00055 & .13007 & -.08874 & .00089 \\
\hline 8.00 & .37798 & -.12465 & $.00024_{4}$ & -.12318 & .00026 & .11847 & -.06696 & .00055 \\
\hline 8.50 & .33645 & -.11744 & .00015 & -.11648 & .00018 & .11303 & -.05757 & .00043 \\
\hline 9.00 & .29759 & -.11099 & .00009 & -.11037 & .00012 & .10786 & -.04920 & .00033 \\
\hline 10.00 & .22876 & -.09996 & .00003 & -.09970 & .00005 & .09843 & -.03533 & .00020 \\
\hline 12.00 & .12704 & -.08333 & .0 & -.08329 & .00001 & .08299 & -.01721 & .00007 \\
\hline 14.00 & .06590 & -.07142 & .0 & -.07142 & .0 & .07136 & -.00790 & .00002 \\
\hline 16.00 & .03238 & -.06250 & .0 & -.06250 & .0 & .06248 & -.00346 & .00001 \\
\hline 19.00 & .01031 & -.05263 & .0 & -.05263 & .0 & .05263 & -.00094 & .0 \\
\hline 22.00 & .00306 & -.04545 & .0 & -.04545 & .0 & .04545 & -.00024 & $\therefore$ \\
\hline
\end{tabular}


Table 4 (Contd).

\begin{tabular}{|c|c|c|c|c|c|c|c|c|}
\hline $\mathrm{R}$ & $\mathrm{K}_{\mathrm{A}}$ & $\mathrm{K}_{\mathrm{A}}^{\prime}$ & $K^{\prime}$ & $V_{c}$ & $v_{\text {ex }}$ & $E_{s}$ & $E_{t}$ & $E_{t}-E_{s}$ \\
\hline 3.00 & -.21580 & .00304 & .14094 & .45068 & .13487 & -.28295 & .13877 & .421722 \\
\hline 4.00 & -.19536 & .00282 & .11964 & .22854 & .07097 & -.47071 & -.31270 & .158018 \\
\hline 5.00 & 0.16910 & .00230 & .09107 & .10055 & .01177 & -.58523 & -.50809 & .077146 \\
\hline 6.00 & -.14017 & .00173 & .06531 & .03819 & -.01041 & -.64268 & -.59276 & .049920 \\
\hline 7.00 & -.11177 & .00124 & .04426 & .01206 & -.01434 & -.66566 & -.63001 & .035650 \\
\hline 8,00 &.--8620 & .00085 & .02836 & .00266 & -.01158 & -.67160 & -.64718 & .024415 \\
\hline 8.50 & -.7487 & .00070 & .02224 & .00082 & -.00958 & -.67167 & -.65207 & .019599 \\
\hline 9.00 & -.06460 & .00057 & .01722 & -.00010 & -.00765 & -.67091 & -.65551 & .015399 \\
\hline 10.00 & -.04726 & .00040 & .00996 & -.00059 & -.00449 & -.66863 & -.65967 & .008952 \\
\hline 12.00 & -.02394 & .00016 & .00264 & -.00029 & $=.00184$ & -.66590 & -.66222 & .003683 \\
\hline 14.00 & -.01132 & .00006 & .00067 & -.00008 & -.00045 & -.66432 & -.66342 & .000895 \\
\hline 16.00 & -.00513 & .00002 & .00015 & -.00002 & -.00010 & -.66391 & -.66371 & .000194 \\
\hline 19.00 & -.00146 & .00001 & .00001 & -.000001 & -.00001 & -.66380 & -.66378 & .000017 \\
\hline 22.00 & -.000 & .0 & .0 & .0 & .0 & -.66379 & -.66379 & .000001 \\
\hline
\end{tabular}


The energies of the singlet and triplet states, and their difference are shown in fig. 7. The singlet state shows a shallow minimum at $r=8.3 a_{0}$ whose depth is $0.008 \mathrm{ry}(0.109 \mathrm{eV})$. The triplet state has no minimum in the Heitler-London calculation; however, if a Van der Waals attraction of the form $V_{V D W}=-c / R^{6}$ is added, a minimum is found. The Van der Waals constant $c$ has been deduced from scattering measurements to have the value $\mathrm{c}=3.014 \times 10^{3} \mathrm{ry}^{6} \mathrm{a}^{6} 34$. The minimum in the triplet state energy occurs at $R=15.8 \mathrm{a}_{\mathrm{o}}$, and has a depth of $1.0 \times 10^{-4} \mathrm{ry}$ $(.0013 \mathrm{eV})$. It is not possible to determine the effect of the Van der Wals interaction on the minimum in the singlet state since the simple expression is not valid at that distance.

Dalgarno and Rudge 35 have derived a simple formula for the energy difference between singlet and triplet states at large atomic separations, which they have applied to alkali metal atom pairs. Their formula is

$$
E_{t}(R)-E_{S}(R)=2 \pi S(R) \bar{q}(R / 2) \psi(R / 2)
$$

where $S$ is the overlap integral, $\Phi$ is the wave function for the valence electron bound to one atom and $\psi$ refers to the other atom. These functions are approximated in their paper by the leading term in the asymptotic series for a coulomb potential. Since a number of assumptions are involved in their treatment, it should be noted that for $\mathrm{H}_{2}$, the Dalgarno-Rudge formula constantly underestimates 


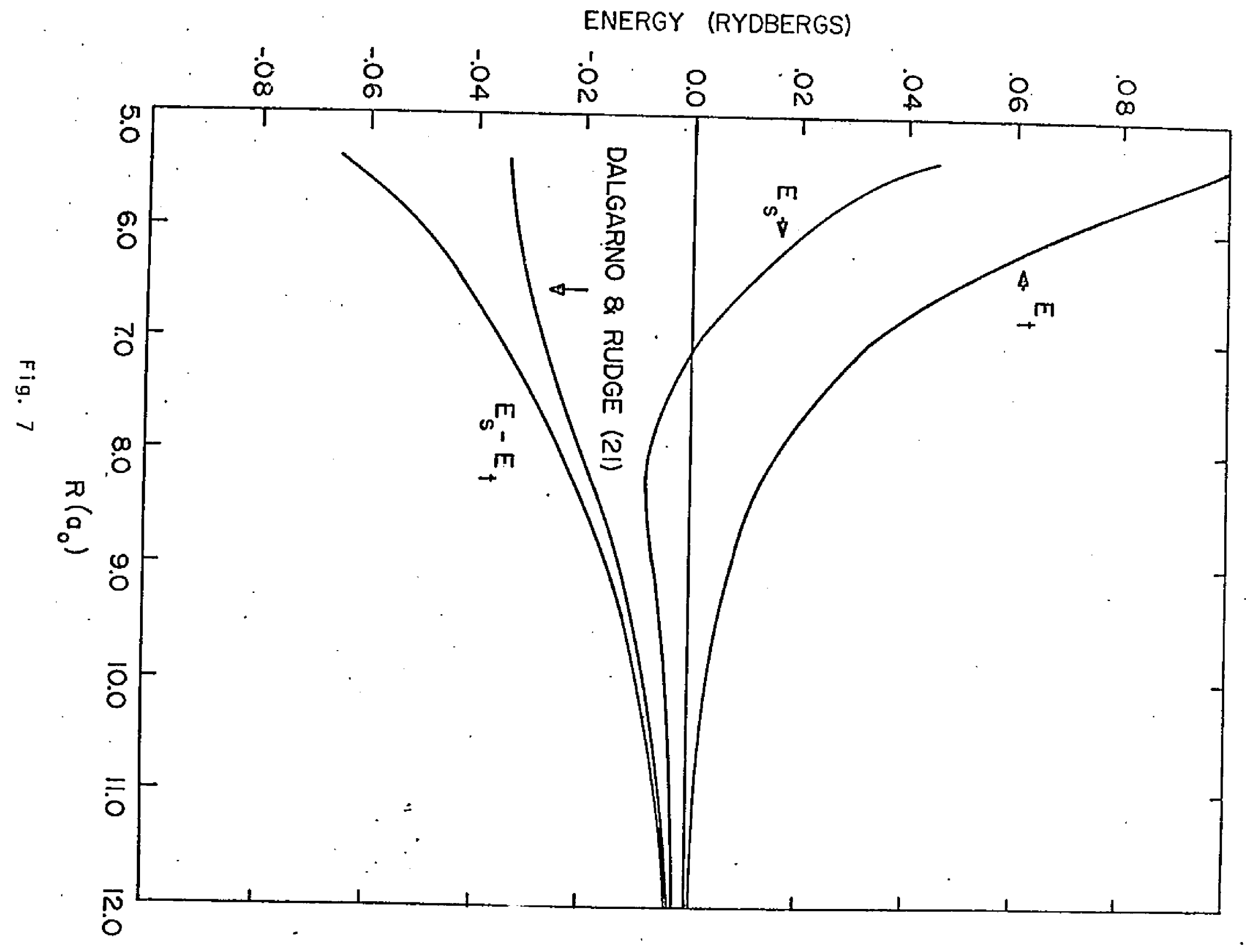


the energy difference between these states by 20 to $30 \%$ at moderately large atomic distances not only with respect to the Heitler-London resuits, but also with regard to the asymptotic formula of Herring and Flicker ${ }^{30}$ and the variational calculation of Kolos and Wolniewicz. ${ }^{36}$ Consequently, its use in the spin exchange calculations is open to some question, although it appears from this work that the error in the cross section is not large. When $(4,1)$ is evaluated using four term asymptotic coulomb wave functions for $\mathrm{Na}$ and $\mathrm{Cs}$ it gives a smaller energy difference than is yielded by the HeitlerLondon calculation by about 10 to $20 \%$ in the range of distances important in the spin exchange problem. However, if errors of this magnitude can be tolerated, the Dalgarno-Rudge formula is quite convenient since overiap integrals are much easier to compute than two electron coulomb and exchange integrals. The result of the Dalgarno-Rudge computation is compared with the present result in fig. 7 .

The present results do not agree well with indications from experimental scattering measurements that the minimum in the triplet state potential energy curve occurs in the vicinity of $9 a_{0} 37,38$ The present experimental results are summarized in table 5 . The reason for the discrepancy in the triplet state is not known, and appears to be difficult to determine, since the Heitler-London calculation should be accurate at this distance. 
Table 5. Position of minimum and binding energies for singlet and triplet states of the $\mathrm{Na}-\mathrm{Ca}$ system

\begin{tabular}{|c|c|c|c|c|}
\hline & $E_{s}^{m}(r y)$ & $r_{s}^{m}\left(a_{o}\right)$ & $E_{t}^{m}(r y)$ & $r_{t}^{m}\left(a_{0}\right)$ \\
\hline $\begin{array}{l}\text { Neumann } \\
\text { and Pauly } \\
\text { (ref. } 38 \text { ) }\end{array}$ & $5.07 \times 10^{-2}$ & 4.91 & $1.25 \times 10^{-3}$ & 9.3 \\
\hline $\begin{array}{l}\text { Pritchard et al. } \\
\text { (ref. } 37)\end{array}$ & & & $1.96 \times 10^{-3}$ & 8.55 \\
\hline Present work & $8.0 \times 10^{-3}$ & 8.28 & $1.0 \times 10^{-4}$ & 15.8 \\
\hline $\begin{array}{l}\text { Dalgarno and } \\
\text { Rudge } \\
\text { (ref. 35) }\end{array}$ & & & $.98 \times 10^{-4}$ & 15.8 \\
\hline
\end{tabular}


VI SPIN EXCHANGE CROSS SECTIONS FOR Na - CS

Denote, as before, the sodium and cesium atoms by $A$ and $B$, respectively. Let the spin I of ion $A$ be coupled to the spin of the valence electron to form a resultant total spin $F$. That is, $F$ is the total spin quantum number of atom $A$ before the collision. We are interested in the process in which the total spin of atom $A$ is changed to $F^{\prime}$ such that $F^{\prime} \neq F$. This is accomplished by flipping the spin of its valence electron. If, for example, A denoted a $H$-atom, we would have the process $F=1 \rightarrow F^{\prime}=0$ or the reverse process $F=0 \rightarrow F^{\prime}=1$.

A quantum formulation of the problem was given by Dalgarno 39 for $\mathrm{H}-\mathrm{H}$ collisions and attempts to improve approximate calculations within this formulation followed 40,41 . The quantal formulation was subseguently extended to include other more complex systems 35,42 , including most alkali metal pairs. Dalgarno and Rudge ${ }^{35}$ obtain for the total spin change cross section, which is what one would observe in an actual experiment,

$$
Q\left(F, F^{\prime}\right)=\frac{\pi\left(2 F^{\prime}+1\right)}{2 k^{2}(2 l+1)} \sum_{l=0}^{\infty}(2 l+1) \sin ^{2}\left(\eta_{l}^{s}-\eta_{l}^{t}\right)
$$

where $k$ is the wave number of relative motion, and $\eta_{\ell}^{s}$ and $\eta_{\ell}^{t}$ are the singlet and triplet phase shifts. A reduced spin exchange cross section, $Q_{e x}$, independent of $F^{\prime}$ and 1 can be defined by the equation

$$
\mathrm{Q}_{\mathrm{ex}}=\frac{\pi}{\mathrm{k}^{2}} \stackrel{\infty}{l}=0_{0}(2 \ell+\mathrm{I}) \sin ^{2}\left(\eta_{l}^{\mathrm{s}}-\eta_{l}^{\mathrm{t}}\right)
$$


so that

$$
Q\left(F, F^{\prime}\right)=\frac{\left(2 F^{\prime}+1\right)}{2(21+1)} Q_{e x}
$$

In heavy particle collisions the number of phase shifts contributing to the cross section is large, and we employ the semi-classical approximation which consists in replacing the summation in (6.1) by an integral over impact parameters $p$, where $k p=\ell+\frac{1}{2}$, and in using the JWKB approximation ${ }^{14}$ to evaluate the phase shifts. The expression for the reduced cross section then becomes

$$
Q_{e x}=2 \pi \int_{0}^{\infty} p \sin ^{2}\left\{\frac{1}{v} f(p)\right\}
$$

where

$$
f(p)=\int_{0}^{\infty} \frac{E_{t}(R)-E_{s}(R)}{\left(1-p^{2} / R^{2}\right)^{\frac{T}{2}}} d R
$$

and $v$ is the relative velocity of the colliding atoms. Detailed calculations show that the semi-classical approximation is satisfactory in the case of $H-H$ collisions. 40,41 For $E_{t}$ and $E_{s}$ we will use our results from the previous section. The region of interest is that of faitly large interatomic separations and in this region the difference between the triplet and singlet potential energies is very satisfactorily given by the analytic expression 


$$
E_{t}(R)-E_{s}(R)=A R^{5} \exp \left(-\alpha_{0} R\right)
$$

with

$$
\begin{aligned}
A & =0.007925 \\
\alpha_{0} & =1.0983
\end{aligned}
$$

This expansion is an emprirical fit rather than an asymptotic expansion of the integrals. We insert (6.4) into (6.3) and obtain

$$
f(z)=A\left(z / \alpha_{0}\right)^{6} \quad \int_{1}^{\infty} \frac{t^{6} e^{-z t}}{\left(t^{2}-1\right)^{\frac{1}{2}}} d t
$$

where the substitutions

$$
\frac{\mathrm{R}}{\mathrm{p}}=\mathrm{t}, \mathrm{z}=\alpha_{\mathrm{o}} \mathrm{p}
$$

have been made. The integral can be evaluated in terms of Modified Bessel Functions. The algebra, though a little lengthy, is straightforward and gives the result

$$
f(z)=A\left(z / \alpha_{0}\right)^{6}\left\{\left(K_{6}+6 K_{4}+15 K_{2}+10 K_{0}\right) / 32\right\}
$$

Let $\delta(z)=\frac{1}{v} f(z)$; then use of this and (6.6) in (6.2) gives for the cross section

$$
Q_{e x}=\left(2 \pi / \alpha_{0}^{2}\right) \int_{0}^{\infty} z \sin ^{2} \delta(z) d z
$$


We evaluate (6.7) in the following way. First we calculate $f(z)$, or for a given value of initial relative velocity $\nu, \frac{1}{\nu} f(z)$. For not too large values of $z, \delta(Z)$ is quite large and $\sin ^{2} \delta(z)$ oscillates rapidly. For large values of $z, \delta(z)$ goes uniformly to zero as $z \rightarrow \infty$ and so does $\sin ^{2} \delta(Z)$. We break up the integral (6.7) into two parts:

$$
Q_{e x}=\left(2 \pi / \alpha_{0}^{2}\right)\left\{\int_{0}^{z_{0}} z \sin ^{2} \delta(z) d z+\int_{z_{0}}^{\infty} z \sin ^{2} \delta(z) d z\right\}
$$

$z_{0}$ is chosen by actually looking at the function $\sin ^{2} \delta(z)$, and it is that value such that for $z<z_{0}, \sin ^{2} \delta(z)$ oscillates rapidly between values of zero and one so that we can replace it by its average value of $1 / 2$. The second term is integrated numerically by actually calculating the modified Bessel functions entering $\delta(z)$ through (6.6). In all cases the $z_{0}$ chosen was a little smaller than the value of $Z$ for which $\sin ^{2} \delta(Z)$ ceases to oscillate. (This occurs when the phase $\delta$ first attains the value of $\pi / 2$ ). Values of $z_{0}$ ranged between $15 a_{0}$ and $12 a_{0}$, slowly decreasing with increasing relative velocity. It is, therefore, the region of interatomic separations of about $12 a_{0}$ and greater that are significant in the problem and here the analytic fit to $E_{t}-E_{s}$ given by $(6.4)$ is very good.

lit is convenient to characterize the relative velocity by an effective temperature $T$ defined through

$$
\frac{1}{2} \mu v^{2}=K T
$$


where $\mu$ is the reduced mass of the colliding pair and $k$ is Boltzmann's constant. In fig. 8 we show the behaviour of the phase shift, $\delta$, and $\sin ^{2} \delta$ as functions of $Z$ for a speed corresponding to a temperature of $500^{\circ} \mathrm{K}$.

Firsov ${ }^{43}$ has suggested the following approximation to $Q_{e x}$ given by $(6,2)$.

$$
Q_{e x}=\frac{1}{2} \pi *^{2}
$$

where $p *$ is that value of $p$ for which

$$
\delta(p *)=1 / \pi
$$

This approximation has been extensively used to avoid numerical integrations. It is interesting to check the accuracy of this method. In the present case Firsov's approximation predicts a cross section of $1.46 \times 10^{-14} \mathrm{~cm}^{2}$ for a relative velocity of $6.51 \times 10^{4} \mathrm{~cm} / \mathrm{sec}$. This is lower than our result for the same velocity by less than $3 \%$. The reduced sp in exchange cross sections calculated for collisions of $\mathrm{Na}$ and $\mathrm{CS}_{\mathrm{s}}$ through equation (6.7) are given in table 6.

Using (6.8) as the defining equation for $T$ we can then obtain the following approximate analytic expression for $Q_{\text {ex }}$ vs. $T$.

$$
Q_{e x}=a-b \ln T
$$

with $T$ measured in degrees Kelvin. The constants $a$ and $b$ were found to be

$$
a=2.106, b=0.100
$$




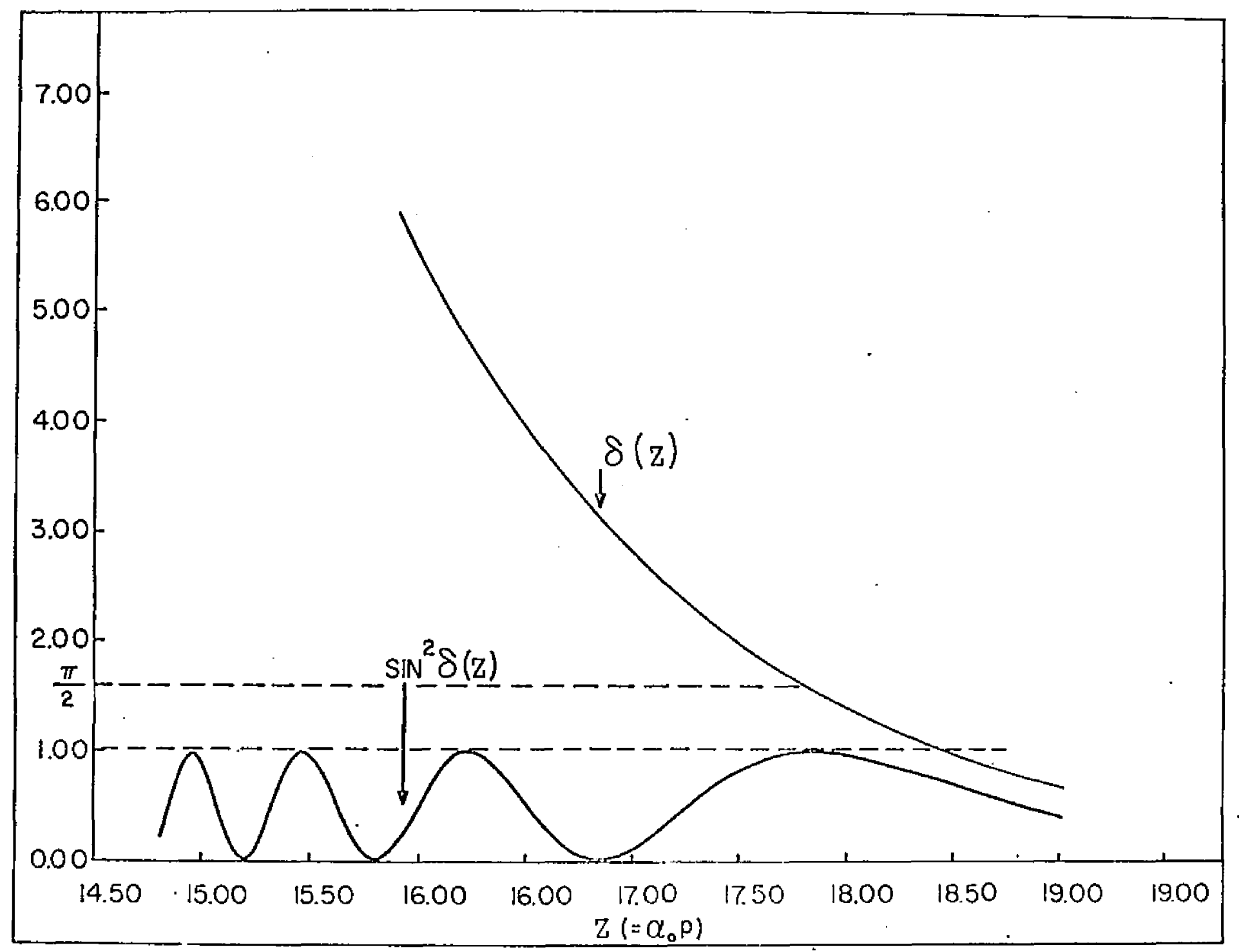

Fig. 8 
Table 6. Reduced spin exchange cross sections for $\mathrm{Na}-\mathrm{Cs}$ collisions as a function of relative velocity.

\begin{tabular}{|c|c|c|}
\hline \multicolumn{2}{|c|}{ relative velocity } & \multirow{2}{*}{$\begin{array}{l}\text { cross sections } \\
\text { (in units of } 10^{-14} \mathrm{~cm}^{2} \text { ) }\end{array}$} \\
\hline$\left(\operatorname{in}^{\circ} \mathrm{K}\right)$ & $($ in $\mathrm{cm} / \mathrm{sec})$ & \\
\hline 50 & $2.06 \times 10^{4}$ & 1.72 \\
\hline 100 & 2.91 & 1.65 \\
\hline 200 & 4.12 & 1.58 \\
\hline 300 & 5.05 & 1.54 \\
\hline 400 & 5.82 & 1.51 \\
\hline 500 & 6.51 & 1.49 \\
\hline 600 & 7.14 & 1.47 \\
\hline 700 & 7.71 & 1.45 \\
\hline 800 & 8.24 & 1.44 \\
\hline 200 & 8.74 & 1.43 \\
\hline 1000 & 9.21 & 1.42 \\
\hline 1200 & 10.09 & 1.40 \\
\hline 1500 & 11.28 & 1.38 \\
\hline 3000 & 15.95 & 1.31 \\
\hline 10000 & 29.13 & 1.20 \\
\hline
\end{tabular}


in units of $10^{-14} \mathrm{~cm}^{2}$.

The normalized Maxwellian distribution of the relative velocities of the colliding particles is

$$
m(v)=(\mu / 2 \pi K \theta)^{3 / 2} e^{-\frac{1}{2} \mu v^{2} / K \theta}
$$

where $\theta$ is the temperature of the system. We calculate an effective cross section, $Q_{e f f}$, defined by

$$
Q_{e f f}=\int m(v) Q_{e x}(v) d^{3} v
$$

The calculation is straightforward and yields

$$
Q_{\text {eff }}=a-b(2-\ln (4 \gamma / \theta))
$$

where $\ln \gamma=.57722$ is Euler's constant. In fig. 9 we plot $Q_{\text {eff }}$ as a function of the temperatue $A$ of the system. 


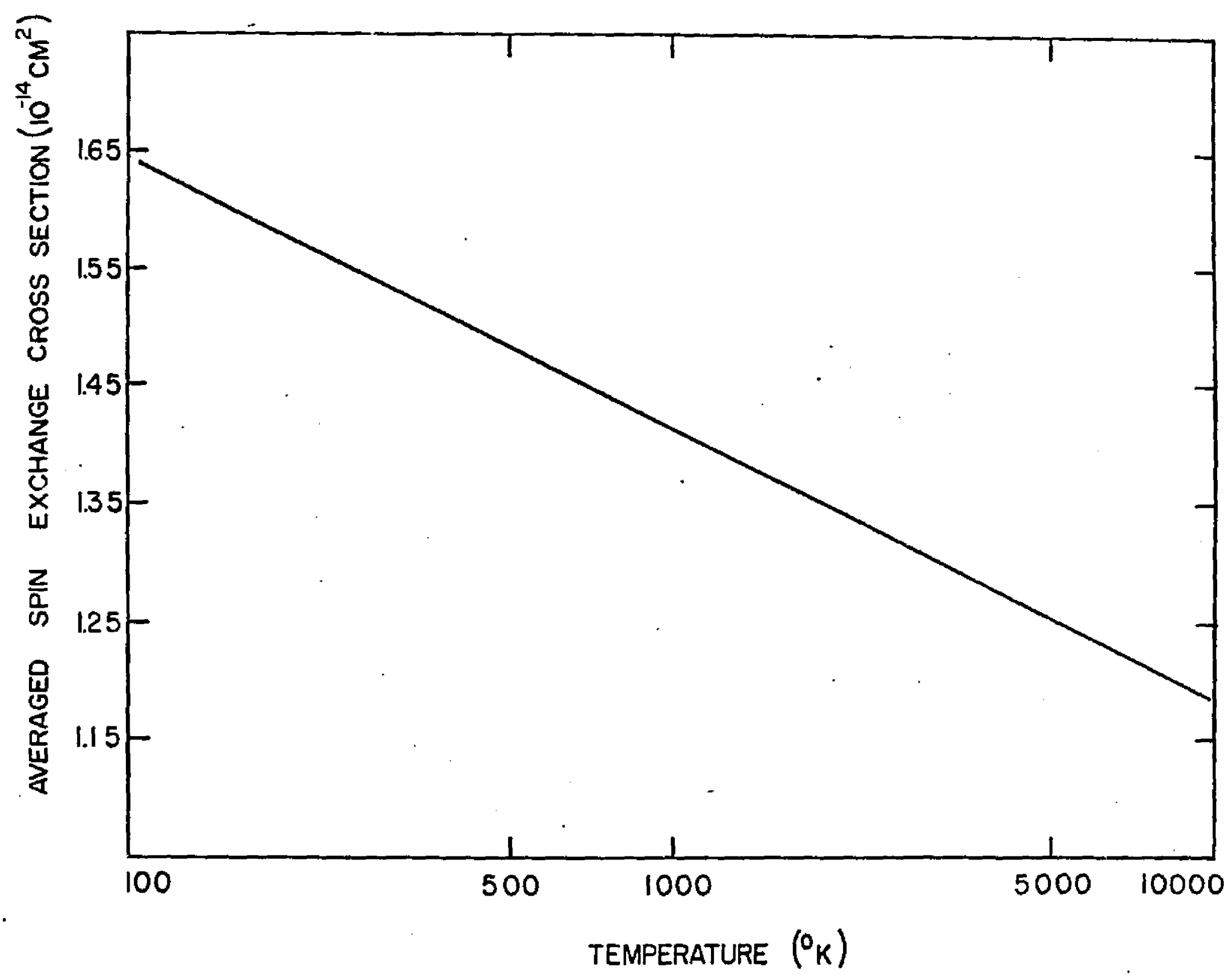

Fig: 9 


\section{SUMMARY AND CONCLUSION}

The empirical pseudopotential method provides us with a practical means of treating a many-electron problem. It is simple enough to be used in calculations, and, when the pseudopotential parameters are determined from the free atom energy levels by numerical integration of the wave equation rather than through any simplified variational method, it reproduces fairly accurately the free atom spectroscopic data. Having calculated the pseudopotential for sodium and cesium, we have used it in a Heitler-London calculation to obtain potential energy curves for the lowest singlet and triplet levels of the $\mathrm{Na}$ - Cs system. The resulting energy curves were in turn used to calculate spin exchange cross sections.

The effects of elastic scattering, (deviations from straight line trajectory), by a Van der Waals potential, have been considered and our principal conclusion is that inclusion of elastic scattering will not significantly alter the calculated cross sections for excitation under normal circumstances.

It will probably be worthwhile to use the pseudopotential for calculations of potential energy curves associated with homopolar molecules and then obtain macroscopic properties of the gas (e.g. viscosities, virial coefficients, etc.) which can be compared with experimental results which are abundant. This comparison will tell how good the potential energy curves are, and in turn, serve as a measure of the accuracy of the pseudopotential. 
In addition, it has become recently possible with crossed beams experiments to make direct measurements of differential scattering cross sections. This, then, would be interesting to formulate theoretically. 


\section{REFERENCES}

1. J. Call laway and E. Bauer, Phys. Rev. 140A, 1072(1965).

2. J. Callaway and A. F. Dugan, Phys. Rev. 163, 26(1967).

3. J. Callaway and P. S. Laghos, Phys. Letters, 26A, 394(1968).

4. J. 0. Hirshfelder, C. F. Curtiss, and R. B. Bird, 'Molecular Theory of Gases and Liquids', John Wiley \& Sons Inc., N. Y. 1954.

5. S. Chapman and T. G. Cowl ing, 'The Mathematical Theory of Non-uniform Gases', 2nd Ed., Cambridge Univ. Press, London and N. Y. 1952 .

6. F. London, Z. Phys. Chem, 11B, 222(1930)

7. E. A. Guggenheim and M. L. McGlashan, Proc. Roy. Soc. (London) $\underline{225 A}, 456(1960)$.

8. Y P Varshni, Revs. Mod. Phys. 29, 664 (1957).

9. R. A. Buckingham, Planet. Space Sci. 3, 205(1961).

10. A. A. Abrahamson, Phys. Rev. 130, 693(1963).

11. H. Pauly and \$. P. Townes in 'Advances in Atomic and Molecular Physics', vol. 1(1965), Academic Press.

12. V. P. Leonas and E. V. Samuılov, High Temp. 4, 664(1966).

13. J. 0. Hirscfelder and E. P. Wigner, J.Chem. Phys. Z, 616(1939).

14. K. Ford and J. A. Wheeler, Ann. Phys. (N.Y.) 7, 259(1959)

15. J. C. Phillips and L. Kieinman, Phys. Rev. 116, 287(1959)

16. H. Hellmann, Acta. Phys icochimica URSS 1, 913(1934).

17. H. Preuss, Z. Naturforsch 10A, 365(1955).

18. L. Szasz and G. McGinn, J. Chem. Phys, 42, 2363(1965). 
19. G. J. lafrate, J. Chem, Phys. 46, 729(1967).

20. H. Hellmann and W. Kassatotschkin, J. Chem. Phys. 4, 234(1936); Acta. Physicochimica URSS $\underline{5}, 23(1936)$.

21. K. Ladanyi, Acta. Phys, Hung. 5, 361)1956).

22. J. Callaway, Phys. Rev. 112, 322(1958).

23. A. J. Hughes and J. Callaway, Phys. Rev. 136A, 1390(1964).

24. N. W. Ashcroft, J. Phys. C. 1, 232(1968).

25. B. P. Zapol, P. E. Kunin, and I. M. Taksar, Optics and Spectroscopy $23,191(1967)$.

26. P. Gomabs, Pseudopotentiale (Springer-Verlag, Vienna, N. Y., 1967).

27. W. E. Bayliss, to be published.

28. L. Biermann and K. Lubeck, Z. Astrophys. 25, 325(1948).

29. W. Heitler and F. London, Z Physik 44, 455(1927).

30. C. Herring and M. Flicker, Phys. Rev. 134A, 362(1964).

31. For a detailed look at the Heitler-London method, as originally given by Heitler and London, along with various subsequent modifications to it when applied to the $\mathrm{H}_{2}$-molecular problem see J. C. Slater, 'Quantum Theory of Molecules and Solids', vol. 1, ch. 3(1963)

32. Ф. Barrau, Kgl. Danske Videnskab. Selskab, Mat. fys. Medd. Z, $14(1927)$.

33. The usefulness of these coordinates in molecular integrais has been very great and a lot of formalism has appeared in the literature see, for example, Alf Lofthus, Mol. Phys, 5, 105(1962); 6, $115(1963) ; 13,461(1967)$; and references therein. Many of 
these integrals have been tabulated and, furthermore, at present there exist standard molecular (computer) programs.

34. V. Buck and H. Pauly, Z. Physik 185, 155(1965).

35. A. Dalgarno and M.R.H. Rudge, Proc. Roy. Soc, A286, 519(1965).

36. W. Kolos and L. Woiniewicz, J. Chem, Pjys. 43, 2429(1965).

37. D. E. Pritchard, D. C. Burnham, and D. Kleppner Phys. Rev. Letters $\underline{19}, 1363(1967)$.

38. W. Neumann and H. Pauly, Phys, Rev. Letters 20, 357(1968).

39. A. Dalgarno, Proc, Roy. Soc. A262, 132(1961).

40. F. J. Smith, Planet. Sp. Sci. 11, 1126(1963).

41. A. Dalgarno and R. W. Henry, Proc. Roy. Soc. 83, 157(1964).

42. F. J. Smith, Planet. Sp. Sci, 14, 937(1966); 14, 927(1966).

43. 0. Firsov, Zh. Eks. Teor. Fiz. 21, 1001 (1951) 


\section{VITA}

Panayiot is Sophocleous Laghos was born January 31, 1940, in Meniko, Nicosia, Cyprus. After attending local schools he completed his high school education at the English School Nicosia in 1958. He then held a government job at the office of the Commissioner of Nicosia, Cyprus, unti] September 1960 when he enrolled at the American University of Beirut and recelved his B.Sc. degree in Physics 1964. He entered the Graduate School at Louisiana State University in the Department of Physics and Astronomy, in the fall of 1964, and is presently a candidate for the degree of Doctor of Philosophy. 
Candidate: Panayiotis Sophocleous Laghos

Major Field: $\quad$ Physics

Title of Thesis: Application of the Pseudopotential Method to Atomic Scattering

Approved:

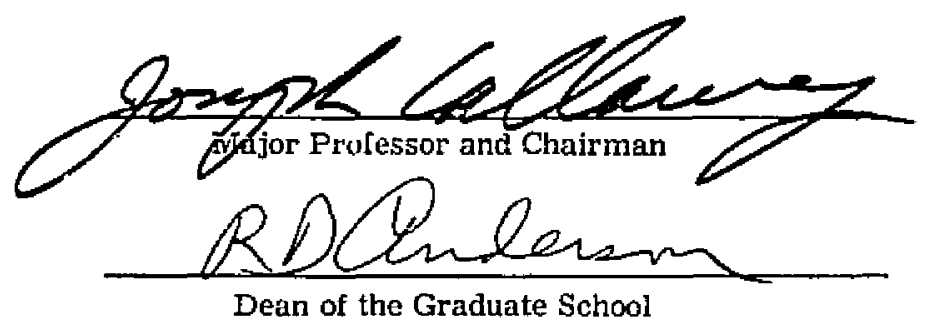

EXAMINING COMMITTEE:

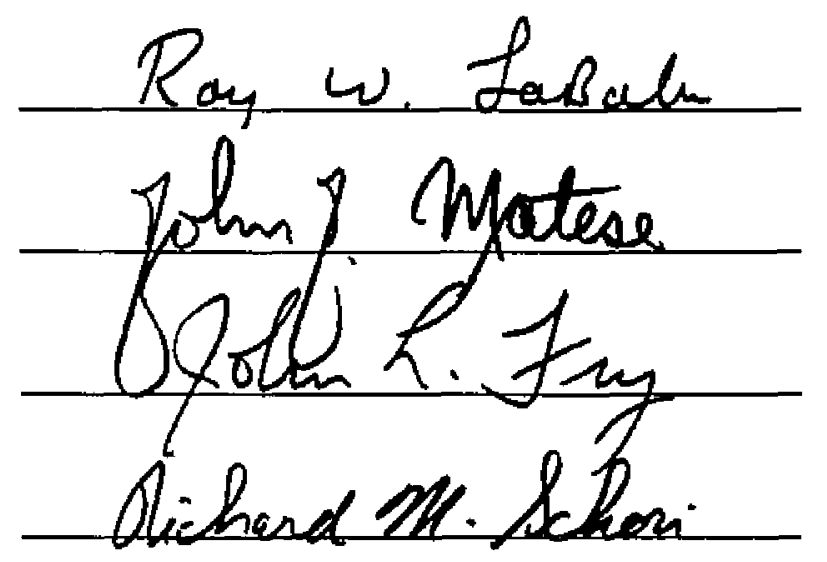

Date of Examination:

May 12,1969 